\title{
Discrete-time model for a substance motion in a channel of a network. Application to a human migration channel
}

\author{
Kaloyan N. Vitanov, Nikolay K. Vitanov ${ }^{1,2 *}$ \\ ${ }^{1}$ Institute of Mechanics, Bulgarian Academy of Sciences, Acad. \\ G. Bonchev Str., Block 4, 1113 Sofia, Bulgaria \\ 2 Max-Planck Institute for the Physics of Complex Systems, \\ Noethnitzerstr. 38, 0187 Dresden, Germany
}

\begin{abstract}
We discuss a discrete-time model for motion of substance in a channel of a network. For the case of stationary motion of the substance and for the case of time-independent values of the parameters of the model we obtain a new class of statistical distributions that describe the distribution of the substance along the nodes of the channel. The case of interaction between a kind of substance specific for a node of the network and another kind of substance that is leaked from the channel is studied in presence of possibility for conversion between the two substances. Several scenarios connected to the dynamics of the two kinds of substances are described. The studied models: (i) model of motion of substance through a channel of a network, and (ii) model of interaction between two kinds of substances in a network node connected to the channel, are discussed from the point of view of human migration dynamics and interaction between the population of migrants and the native population of a country.
\end{abstract}

\section{Introduction}

Nonlinear dynamics of complex systems is studied much in the last decades [1] - [19] and special attention was set on the areas of social dynamics and population dynamics [20]- 32].

*corresponding author: vitanov@imbm.bas.bg 
In the last decades models of flows in networks are much used in the study of different kinds of problems, e.g, transportation problems [33]-39]. In the course of the years the research interest (that initially was focused on problems such possible maximal flows in a network, minimal cost flow problems, or meeting fixed schedule with minimum number of individuals) expanded to the research areas of: just in time scheduling, shortest path finding, self-organizing network flows, facility layout and location, modeling and optimization of scalar flows in networks [40], optimal electronic route guidance in urban traffic networks [41, isoform identification of RNA [42, memory effects 43, etc. (see, e.g., 44] - 52]). We shall discuss in this article a discrete - time model for the motion of a substance through a network channel in presence of possibility for "leakage" of substance. One possible application of the discussed model is for the flow of a substance through a channel with use of part of the substance in some industrial process in the nodes of the channel. However the model has more possible applications and we shall show this for the case of human migration flow. Human migration is an actual research topic that is very important for taking decisions about economic development of regions of a country [53] - 62]. Human migration is closely connected, e.g., to: (i) migration networks [63], 64]; (ii) ideological struggles [27], 28]; (iii) waves and statistical distributions in population systems [25] - 31. We note that the probability and deterministic models of human migration are interesting also from the point of view of applied mathematics [65] - [77].

The text below is organized as follows. In Sect.2 we discuss a discrete - time model for motion of substance in a channel containing finite number of nodes. A class of statistical distributions is obtained in Sect. 3. These distributions describe the distribution of the substance in the nodes of the channel for the case of stationary motion of substance through the channel. Particular cases of the distributions obtained in Sect.3 and in Appendix A are the distributions of Waring, Yule-Simon, and Zipf . In Sect. 4 we study the interaction between two kinds of substances in a node of the network. The substances are: (i) substance that is "native" for the node of the network, and (ii) substance that "leaks" from the corresponding node of the channel to to studied node of the network. In Sect. 5 we apply the the results from Sect. 4 to the case of interaction between population of migrants (the number of migrants may increase by inflow of migrants from the migration channel) and native population of a country. Several concluding remarks are summarized in Sect. 6. Appendix A contains results for the class of statistical distributions that describe the distribution of substance along the nodes of the channel for the case of infinite length of the studied channel. 


\section{Mathematical formulation of the model}

Let us consider a network consisting of nodes connected by edges. We assume existence of a channel in this network - Fig. 1. The structure of the channel

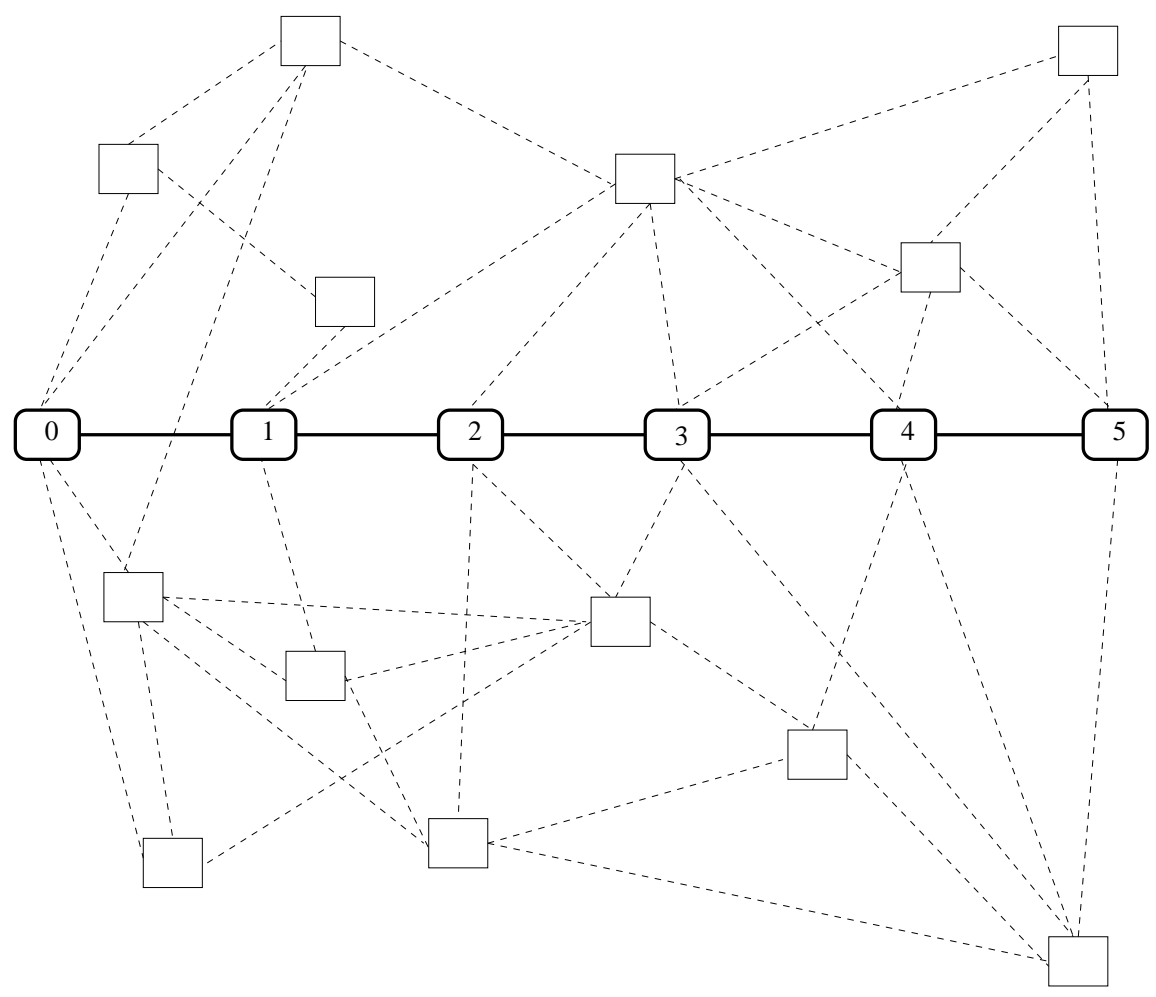

Figure 1: A network and a channel. The channel consists of 6 nodes labeled from 0 to 5 . The node 0 is the entry node of the channel (the substance enters the channel through this node). The nodes and the edges of the channel are marked by bold lines. The other nodes and edges of the network are represented by rectangles and dashed lines.

and it relation to the network are as follows. Several of the nodes of the network together with the corresponding edges belong also to the channel. In Fig. 1 these nodes and edges are marked by bold lines. An exchange of substance between the channel and the network may happen in the nodes of the channel (denoted as "leakage" below). We shall assume that the processes in the network don't influence the flow of the substance in the channel. The "leakage" of the substance from a node of the channel however may influence the processes in the corresponding node of the network. We shall discuss such an influence in Sect. 4 .

We assume further that the channel consists of a chain of $N+1$ nodes 
(labeled from 0 to $N$ ) connected by corresponding edges. Each edge connects two nodes and each node is connected to two edges except for the 0-th node and $N$-th node that are connected by one edge. We assume that a substance can move through the channel. The substance enters the channel through the 0 -th node and moves through the channel. The time is discrete and consists of equal time intervals. At each time interval the substance in a node of the channel (we shall call these nodes also cells below in the text) can participate in one of the following three processes: (a) the substance remains in the same cell and stays in the channel; (b) the substance moves to the next node (i.e., the substance moves from the node $m$ to the node $m+1$ ); (c) the substance"leaks" from the channel: this means that the "leaked" substance doesn't belong anymore to the channel. Such substance may spread through the network. In order to obtain intuition about the process of leaking let us consider a migration channel (this example will be discussed in more detail in Sect. 4 and Sect. 5). The network in this case is a network of countries connected by roads (e.g., the network of European countries). The channel consists of several countries connected by corresponding roads. There is an entry country of the channel and there is a last country (sometimes called the final destination country) of the channel. What moves in this channel are migrants. They move in the direction from the entry country of the channel to the final destination country. In a time interval the migrants: (a) may stay in some of the countries with an intention to move to the next country of the channel.; (b) may move from one country of the channel to the next country of the channel; or (c) may "leak" from the channel for some reason (e.g., they may have obtained permission to stay in the corresponding country of the channel).

Let us formalize mathematically the above considerations. The following processes can be observed in a node of the studied channel:

- exchange (inflow and outflow) of substance with the previous node of the channel (for the nodes $1, \ldots, N$-th of the channel);

- exchange (outflow and inflow) of substance with the next node of the channel (for the nodes $0, \ldots, N-1$ of the channel;

- exchange (inflow and outflow) of substance with the environment of the network;

- "leakages": exchange (outflow and inflow) of substance between the node of the channel and the the correspondent node of the network .

We consider discrete time $t_{k}, k=0,1,2, \ldots$ Let us denote the amount of the substance in the $i$-th node of the channel at the beginning of the 
time interval $\left[t_{k}, t_{k}+\Delta t\right]$ as $x_{i}\left(t_{k}\right)$. For the processes happening in this time interval in the $n$-th node of the channel we shall use the following notations

- $i_{n}^{e}\left(t_{k}\right)$ and $o_{n}^{e}\left(t_{k}\right)$ are the amounts of inflow and outflow of substance from the environment to the $n$-th node of the channel (the upper index $e$ denotes that the quantities are for the environment);

- $o_{n}^{c}\left(t_{k}\right)$ is the amount of outflow of substance from the $n$-th node of the channel to the $(n+1)$-th node of the channel (the upper index $c$ denotes that the quantities are for the channel);

- $i_{n}^{c}\left(t_{k}\right)$ is the amount of the inflow of substance from the $(n+1)$ node of the channel to the $n$-th node of the channel;

- $o_{n}^{n}\left(t_{k}\right)$ and $i_{n}^{n}\left(t_{k}\right)$ are the amounts of outflow and inflow of substance between the $n$-th node of the channel and the corresponding node of the network (the upper index $n$ denotes that the quantities are for the network).

For the entry node of the channel (the 0 -th node) we have exchange of substance with the environment (inflow and outflow); exchange of substance with the next node of the channel (inflow and outflow) and "leakage" of substance from the channel. Thus the change of the amount of substance in the 0 -th node of the channel is described by the relationship

$$
x_{0}\left(t_{k+1}\right)=x_{0}\left(t_{k}\right)+i_{0}^{e}\left(t_{k}\right)-o_{0}^{e}\left(t_{k}\right)-o_{0}^{c}\left(t_{k}\right)+i_{0}^{c}\left(t_{k}\right)-o_{0}^{n}\left(t_{k}\right)+i_{0}^{n}\left(t_{k}\right)
$$

For the nodes of the channel numbered by $i=1, \ldots, N-1$ there is exchange with the environment, "leakage" to the network and exchange with $(i-1)$ st and $(i+1)$-st node of the channel. Thus the change of the amount of substance in the $i$-th node of the channel is described by the relationship

$$
\begin{array}{r}
x_{i}\left(t_{k+1}\right)=x_{i}\left(t_{k}\right)+i_{i}^{e}\left(t_{k}\right)-o_{i}^{e}\left(t_{k}\right)+o_{i-1}^{c}\left(t_{k}\right)-i_{i-1}^{c}\left(t_{k}\right)-o_{i}^{c}\left(t_{k}\right)+i_{i}^{c}\left(t_{k}\right)- \\
o_{i}^{n}\left(t_{k}\right)+i_{i}^{n}\left(t_{k}\right), \quad i=1, \ldots, N-1
\end{array}
$$

For the last node (the $N$-th node of the channel) there is exchange with the environment, "leakage" to the network and exchange with $(N-1)$-st node of the channel. Thus the change of the amount of substance in the $i$-th node of the channel is described by the relationship

$$
\begin{array}{r}
x_{N}\left(t_{k+1}\right)=x_{N}\left(t_{k}\right)+i_{N}^{e}\left(t_{k}\right)-o_{N}^{e}\left(t_{k}\right)+o_{N-1}^{c}\left(t_{k}\right)-i_{N-1}^{c}\left(t_{k}\right)- \\
o_{N}^{n}\left(t_{k}\right)+i_{N}^{n}\left(t_{k}\right)
\end{array}
$$


Eqs.(11) - (3) describe the general case of motion of substance along the channel of the studied network. Below we shall discuss a particular case where no exchange with the environment is present except for the entry node of the channel. In addition we shall assume that:

- there is no inflow of substance from the nodes of the network to the channel.

- there is no outflow of substance from the 0-th node of the channel to the environment

- there is no inflow of substance from the $i$-th node of the channel to the $i$ - 1-th node of the channel, $i=1, \ldots, N$.

For the particular case described above the system of model equations (11) (3) becomes

$$
\begin{gathered}
x_{0}\left(t_{k+1}\right)=x_{0}\left(t_{k}\right)+i_{0}^{e}\left(t_{k}\right)-o_{0}^{c}\left(t_{k}\right)-o_{0}^{n}\left(t_{k}\right) \\
x_{i}\left(t_{k+1}\right)=x_{i}\left(t_{k}\right)+o_{i-1}^{c}\left(t_{k}\right)-o_{i}^{c}\left(t_{k}\right)-o_{i}^{n}\left(t_{k}\right), \quad i=1, \ldots, N-1 \\
x_{N}\left(t_{k+1}\right)=x_{N}\left(t_{k}\right)+o_{N-1}^{c}\left(t_{k}\right)-o_{N}^{n}\left(t_{k}\right)
\end{gathered}
$$

Below we shall study the following particular cases of the quantities from the system of equations (44) - (6)

$$
\begin{aligned}
i_{0}^{e}\left(t_{k}\right) & =\sigma\left(t_{k}\right) x_{0}\left(t_{k}\right) ; \quad o_{0}^{c}\left(t_{k}\right)=f_{0}\left(t_{k}\right) x_{0}\left(t_{k}\right) ; \\
o_{0}^{n}\left(t_{k}\right) & =\gamma_{0}\left(t_{k}\right) x_{0}\left(t_{k}\right) ; \quad o_{i-1}^{c}\left(t_{k}\right)=f_{i-1}\left(t_{k}\right) x_{i-1}\left(t_{k}\right) ; \\
o_{i}^{c}\left(t_{k}\right) & =f_{i}\left(t_{k}\right) x_{i}\left(t_{k}\right) ; \quad o_{i}^{n}\left(t_{k}\right)=\gamma_{i}\left(t_{k}\right) x_{i}\left(t_{k}\right) ; \\
o_{N-1}^{c}\left(t_{k}\right) & =f_{N-1}\left(t_{k}\right) x_{N-1}\left(t_{k}\right) ; \quad o_{N}^{n}\left(t_{k}\right)=\gamma_{N}\left(t_{k}\right) x_{N}\left(t_{k}\right) ;
\end{aligned}
$$

For this particular case the system of equation (44) - (6) becomes

$$
\begin{gathered}
x_{0}\left(t_{k+1}\right)=x_{0}\left(t_{k}\right)+\sigma\left(t_{k}\right) x_{0}\left(t_{k}\right)-f_{0}\left(t_{k}\right) x_{0}\left(t_{k}\right)-\gamma_{0}\left(t_{k}\right) x_{0}\left(t_{k}\right) \\
x_{i}\left(t_{k+1}\right)=x_{i}\left(t_{k}\right)+f_{i-1}\left(t_{k}\right) x_{i-1}\left(t_{k}\right)-f_{i}\left(t_{k}\right) x_{i}\left(t_{k}\right)-\gamma_{i}\left(t_{k}\right) x_{i}\left(t_{k}\right) i=1, \ldots, N-1 \\
x_{N}\left(t_{k+1}\right)=x_{N}\left(t_{k}\right)+f_{N-1}\left(t_{k}\right) x_{N-1}\left(t_{k}\right)-\gamma_{N}\left(t_{k}\right) x_{N}\left(t_{k}\right)
\end{gathered}
$$

We shall study the model equations (81) - (10) in more detail below. 


\section{Distributions of substance corresponding to stationary regime of functioning of the chan- nel}

Below we discuss the model described by Eqs.(8) - (10) for the case when the parameters of the model are time independent (i.e., when $\sigma\left(t_{k}\right)=\sigma$; $\left.\alpha_{i}\left(t_{k}\right)=\alpha_{i}, i=0, \ldots, N ; \gamma_{i}\left(t_{k}\right)=\gamma_{i}, i=0, \ldots, N, f_{i}\left(t_{k}\right)=f_{i}, i=0, \ldots, N\right)$. In this case the system of model equations becomes

$$
\begin{gathered}
x_{0}\left(t_{k+1}\right)=x_{0}\left(t_{k}\right)+\sigma x_{0}\left(t_{k}\right)-f_{0} x_{0}\left(t_{k}\right)-\gamma_{0} x_{0}\left(t_{k}\right) \\
x_{i}\left(t_{k+1}\right)=x_{i}\left(t_{k}\right)+f_{i-1} x_{i-1}\left(t_{k}\right)-f_{i} x_{i}\left(t_{k}\right)-\gamma_{i} x_{i}\left(t_{k}\right) \quad i=1, \ldots, N-1 \\
x_{N}\left(t_{k+1}\right)=x_{N}\left(t_{k}\right)+f_{N-1} x_{N-1}\left(t_{k}\right)-\gamma_{N} x_{N}\left(t_{k}\right)
\end{gathered}
$$

In addition we shall consider the stationary state: $x_{i}\left(t_{k}\right)=x_{i}^{*}$. This stationary state occurs when $x_{i}\left(t_{k+1}\right)=x_{i}\left(t_{k}\right)$ (i.e., there is a motion of substance through the cells of the channel but the motion happens in such a way that the amount of the substance in a given cell remains the same in the course of the time). From the system of equations (11) - (13) we obtain $\left(i=1, \ldots, N-1, x_{0}^{*}\right.$ is a free parameter $)$

$$
x_{i}^{*}=x_{0}^{*} \prod_{j=1}^{i} \frac{f_{j-1}}{f_{j}+\gamma_{j}} ; \quad x_{N}^{*}=x_{0}^{*} \frac{f_{N-1}}{\gamma_{N}} \prod_{j=1}^{N-1} \frac{f_{j-1}}{f_{j}+\gamma_{j}}
$$

The total amount of the substance in the channel is

$$
x^{*}=x_{0}^{*}\left[1+\sum_{k=1}^{N-1} \prod_{j=1}^{k} \frac{f_{j-1}}{f_{j}+\gamma_{j}}+\frac{f_{N-1}}{\gamma_{N}} \prod_{j=1}^{N-1} \frac{f_{j-1}}{f_{j}+\gamma_{j}}\right]
$$

We can consider the statistical distribution $y_{i}^{*}=x_{i}^{*} / x^{*}$ of the amount of substance along the nodes of the channel. $y_{i}^{*}$ can be considered as probability values of distribution of a discrete random variable $\zeta: y_{i}^{*}=p(\zeta=i), i=$ $1, \ldots, N$. For this distribution we obtain

$$
\begin{aligned}
& y_{0}^{*}=\frac{1}{\left[1+\sum_{k=1}^{N-1} \prod_{j=1}^{k} \frac{f_{j-1}}{f_{j}+\gamma_{j}}+\frac{f_{N-1}}{\gamma_{N}} \prod_{j=1}^{N-1} \frac{f_{j-1}}{f_{j}+\gamma_{j}}\right]} \\
& y_{i}^{*}=\frac{\prod_{j=1}^{i} \frac{f_{j-1}}{f_{j}+\gamma_{j}}}{\left[1+\sum_{k=1}^{N-1} \prod_{j=1}^{k} \frac{f_{j-1}}{f_{j}+\gamma_{j}}+\frac{f_{N-1}}{\gamma_{N}} \prod_{j=1}^{N-1} \frac{f_{j-1}}{f_{j}+\gamma_{j}}\right]} ; i=1, \ldots, N-1
\end{aligned}
$$




$$
y_{N}^{*}=\frac{\frac{f_{N-1}}{\gamma_{N}} \prod_{j=1}^{N-1} \frac{f_{j-1}}{f_{j}+\gamma_{j}}}{\left[1+\sum_{k=1}^{N-1} \prod_{j=1}^{k} \frac{f_{j-1}}{f_{j}+\gamma_{j}}+\frac{f_{N-1}}{\gamma_{N}} \prod_{j=1}^{N-1} \frac{f_{j-1}}{f_{j}+\gamma_{j}}\right]}
$$

Eq.(16) describes a class of statistical distributions $\left(f_{i}\right.$ and $\gamma_{i}$ are still not specified). To the best of our knowledge the general form (16) of this class of distributions was not discussed by other authors. Fig. 2 shows several
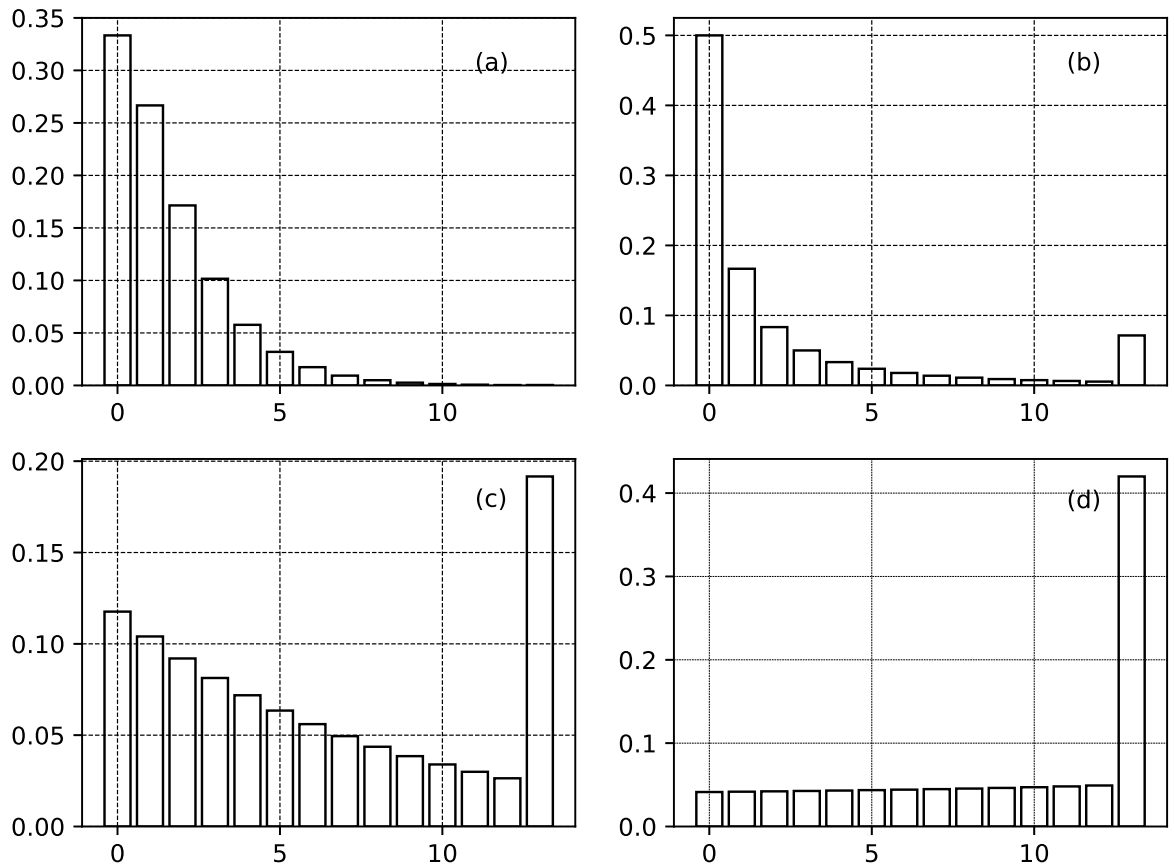

Figure 2: Several examples for distributions from the class of distributions (16) for a channel consisting of 14 nodes. Figure (a): $\gamma_{i}=0.001, f_{i}=$ $0.001 /(i+1)+0.001$. Figure $(\mathrm{b}): \gamma_{i}=0.001, f_{i}=0.001(i+1)$. Figure (c) $\gamma_{i}=0.001, f_{i}=0.0075-0.00002 i$. Figure $(\mathrm{d}): \gamma_{i}=0.00041, f_{i}=$ $0.01-0.0005(i+1)$.

examples for distributions of the class (16). The form of the distribution can be standard as in Fig. 2a but there exist another possible form connected to concentration of substance in the last node of the channel - Figs. 2b,c,d. Fig. $2 \mathrm{~d}$ shows a an interesting form of the distribution that can arise only 
in channel having finite number of nodes: the probability increases with increasing number of the node. We shall discuss again the distributions from Fig. 2 in Sect. 5 where we shall consider the application of the model to channels of human migration.

We note that the class of distributions (16) has interesting particular cases that have been discussed in connection with channels of migration of substance or migration channels of human migration. For and example let $f_{i}=\alpha_{i}+\beta_{i} i, i=1, \ldots, N, \alpha_{i}>0, \beta_{i} \geq 0, \sigma_{0}>0, \gamma_{i} \geq 0$. Then the stationary amount $x_{i}^{*}$ of the substance along the modes of the channel is given by the relationship

$$
\begin{aligned}
x_{i}^{*}= & \frac{\prod_{j=1}^{i}\left[\alpha_{i-j}+(i-j) \beta_{i-j}\right]}{\prod_{j=1}^{i}\left(\alpha_{j}+j \beta_{j}+\gamma_{j}\right)} x_{0}^{*}, i=1, \ldots, N-1 \\
x_{N}^{*}= & \frac{\prod_{j=1}^{N}\left[\alpha_{N-j}+(N-j) \beta_{N-j}\right]}{\gamma_{N} \prod_{j=1}^{N-1}\left(\alpha_{j}+j \beta_{j}+\gamma_{j}\right)} x_{0}^{*}
\end{aligned}
$$

and the statistical distribution connected to this stationary state of functioning of the channel is

$$
\begin{aligned}
y_{0}^{*}= & \frac{1}{1+\sum_{i=1}^{N-1} \frac{\prod_{j=1}^{i}\left[\alpha_{i-j}+(i-j) \beta_{i-j}\right]}{\prod_{j=1}^{i}\left(\alpha_{j}+j \beta_{j}+\gamma_{j}\right)}+\frac{\prod_{j=1}^{N}\left[\alpha_{N-j}+(N-j) \beta_{N-j}\right]}{\gamma_{N} \prod_{j=1}^{N-1}\left(\alpha_{j}+j \beta_{j}+\gamma_{j}\right)}} \\
y_{i}^{*}= & \frac{\prod_{j=1}^{i}\left[\alpha_{i-j}+(i-j) \beta_{i-j}\right]}{\prod_{j=1}^{i}\left(\alpha_{j}+j \beta_{j}+\gamma_{j}\right)} \\
y_{N}^{*}= & \frac{\sum_{i=1}^{N-1} \frac{\prod_{j=1}^{i}\left[\alpha_{i-j}+(i-j) \beta_{i-j}\right]}{\prod_{j=1}^{i}\left(\alpha_{j}+j \beta_{j}+\gamma_{j}\right)}+\frac{\prod_{j=1}^{N}\left[\alpha_{N-j}+(N-j) \beta_{N-j}\right]}{\gamma_{N} \prod_{j=1}^{N-1}\left(\alpha_{j}+j \beta_{j}+\gamma_{j}\right)}}{\frac{\prod_{j=1}^{N}\left[\alpha_{N-j}+(N-j) \beta_{N-j}\right]}{\gamma_{N} \prod_{j=1}^{N-1}\left(\alpha_{j}+j \beta_{j}+\gamma_{j}\right)}}
\end{aligned}
$$


The distribution (18) is a generalization, e.g., of the truncated Waring distribution [79] as well as a generalization of one of distributions discussed in [80. We note that the corresponding distribution for the case of channel of infinite length is a generalization of the Waring distribution and because of this it contains as particular cases several famous distributions such as Zipf distribution or Simon-Yule distribution (see Appendix A). The distribution
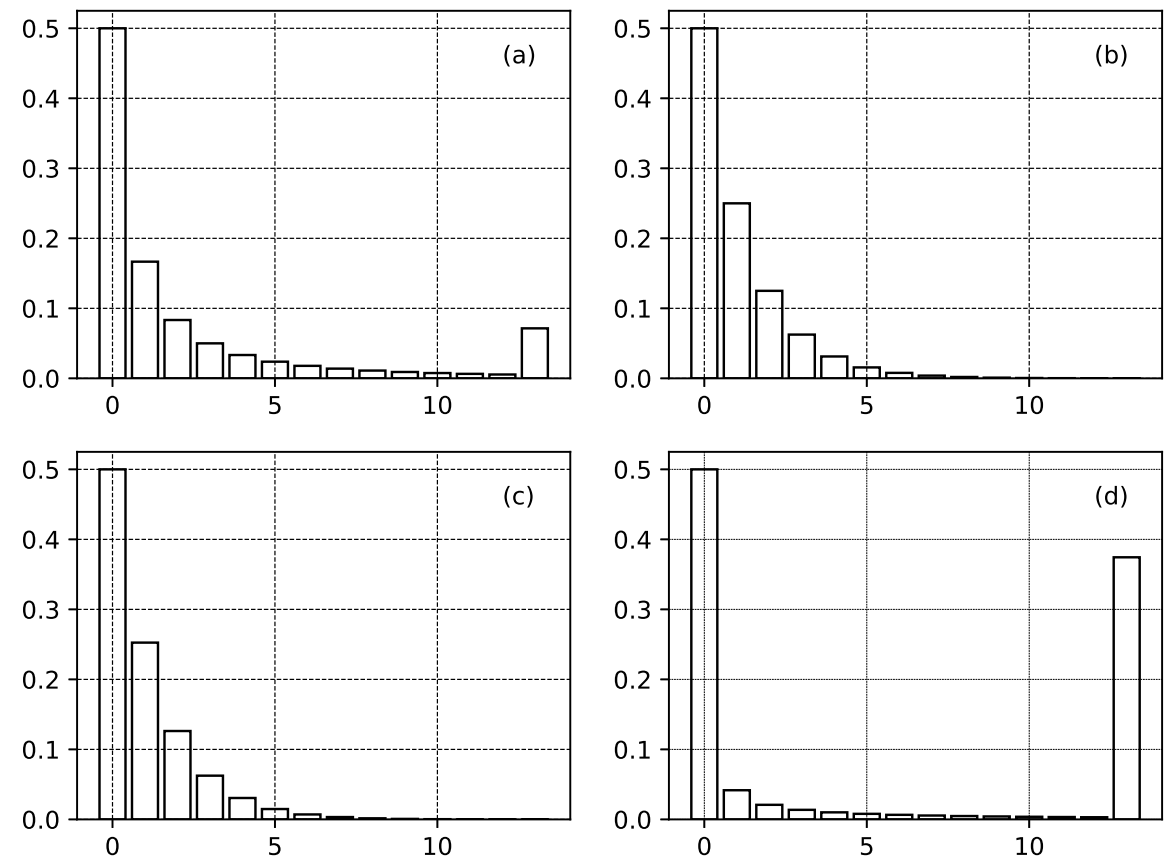

Figure 3: The distribution (18) for a channel consisting of 14 nodes. All parameters $\gamma_{i}$ have the same value $\gamma_{i}=0.001(i=0, \ldots, 13)$ in all figures - 3a, $3 \mathrm{~b}, 3 \mathrm{c}, 3 \mathrm{~d}$. All parameters $\alpha_{i}$ have the same value $\alpha_{i}=0.001(i=0, \ldots, 13)$ in all figures - 3a, 3b, 3c, 3d. Figures show the changes in the form of the distribution when the parameter $\beta_{i}$ is changed. Figure (a): $\beta_{i}=0.001 i$. There is a concentration of the substance in the last node of the channel. Figure (b): $\beta_{i}=0$. The amount of substance in the second half of the channel decreases in comparison to Fig. (a). There is no concentration of substance in the last node of the channel. Figure (c): $\beta_{i}=-0.00002 i$. Amount of substance in the second half of the channel decreases in comparison to the case from Fig. (b). Figure (d): $\beta_{i}=0.01 i$. There is a large concentration of substance in the last node of the channel. 
(18) is visualized in Fig. 3 for fixed values of the parameters $\alpha_{i}$ and $\gamma_{i}$ and for different values of the parameters $\beta_{i}$. As we can see the values of the parameters $\beta_{i}$ influence the situation about the amount of the substance in the second half of the channel. Specific feature of the discussed distributions is the relatively large value of the probability (large value of the amount of substance) in the last node of the channel - Fig. 3a. This probability can increase if the values of $\beta_{i}$ are increased - Fig. 3d. The probability can decrease when the values of $\beta_{i}$ are set to 0 or become negative - Figs. 3b, 3c. Then the tendency is for concentration of substance in the first half of the channel. We shall discuss the distribution (18) below in the text as it describes an interesting situation for the case of channel of human migration, namely the situation where the attractiveness of the countries from the second half of the channel is larger (positive values of $\beta_{i}$ ) (or smaller - negative values of $\beta_{i}$ ) with respect to the attractiveness of the countries from the first half of the channel. Let us note that more information about the corresponding distribution for the case of infinite channel can be obtained from Appendix A.

\section{On interaction between substances in a node that belongs to the channel and to the net- work}

As we have seen above a part of the substance may leave the channel as an outflow ("leakage") from the channel to the corresponding node of the network. Let us study the following problem. We consider a node of the network that contains some amount $A\left(t_{k}\right)$ of substance $A$ and obtains (through its connection with the channel) amount $c\left(t_{k}\right)$ of the substance $B$ in the time interval between $t_{k}$ and $t_{k+1}$. Let us assume presence of two kinds of processes in the discussed node. These processes can: (i) lead to change of the amount of substances $A$ and $B$ without conversion of $A$ to $B$ and $B$ to $A$, and (ii) lead to changes in the amount of the substances $A$ to $B$ by means of conversion of $A$ to $B$ and $B$ to $A$. The model system for the change of the amount of substances in the discussed node will be

$$
\begin{aligned}
& A\left(t_{k+1}\right)=A\left(t_{k}\right)+p\left(t_{k}\right) A\left(t_{k}\right)+q\left(t_{k}\right) A\left(t_{k}\right) B\left(t_{k}\right), \\
& B\left(t_{k+1}\right)=B\left(t_{k}\right)+c\left(t_{k}\right)+r\left(t_{k}\right) B\left(t_{k}\right)-q\left(t_{k}\right) A\left(t_{k}\right) B\left(t_{k}\right),
\end{aligned}
$$

where $p\left(t_{k}\right)$ is the parameter that describes the changes in the amount of the substance $A$ as a result of processes that do not lead to conversion between $A$ and $B ; r\left(t_{k}\right)$ is the parameter that describes the changes in the amount of the 
substance $B$ as a result of processes that do not lead to conversion between $A$ and $B ; q\left(t_{k}\right)$ is parameter that describes the changes as a consequence of the conversion between $A$ and $B$. The stationary state of the system (19) $\left(A\left(t_{k+1}\right)=A\left(t_{k}\right) ; B\left(t_{k+1}\right)=B\left(t_{k}\right)\right)$ is

$$
A^{*}\left(t_{k}\right)=\frac{r\left(t_{k}\right) p\left(t_{k}\right)-c\left(t_{k}\right) q\left(t_{k}\right)}{p\left(t_{k}\right) q\left(t_{k}\right)} ; \quad B^{*}\left(t_{k}\right)=-\frac{p\left(t_{k}\right)}{q\left(t_{k}\right)}
$$

Let us first assume that the parameters $c, p, q, r$ don't depend on time. Fig.
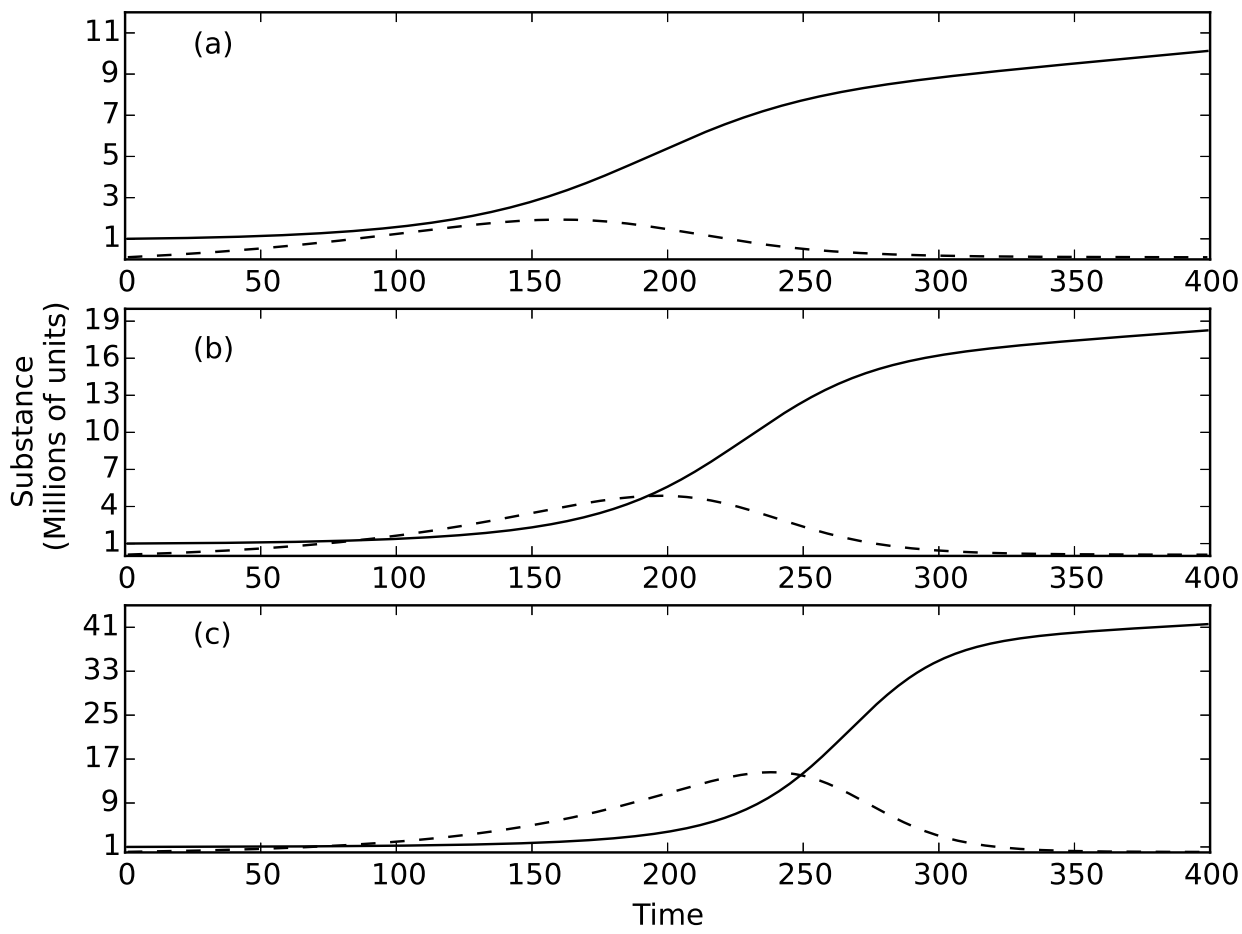

Figure 4: Influence of parameter $q$ for the case of constant values of the parameters in Eqs. (19). Solid line: amount of substance $A$. Dashed line: amount of substance $B$. Figure (a): $q=7 \cdot 10^{-9}$. Figure (b): $q=4 \cdot 10^{-9}$. Figure (c): $q=2 \cdot 10^{-9}$. The values of the other parameters are: $p=5 \cdot 10^{-4}$, $r=2 \cdot 10^{-2}, c=5 \cdot 10^{3}$. The initial conditions are: $A(0)=10^{6}, B(0)=10^{5}$.

4 shows the influence of the parameter $q$ of the amounts of the substances and describes Scenario No.1: Limitation of the amount of substance B by conversion. Larger values of $q$ mean that larger amount of the substance $B$ is converted to substance $A$. The other parameters are chosen in such a way 
that the rate of increase of substance $B$ is larger than the rate of increase of the substance $A$ and in addition the substance $B$ increases also by means of some substance that arrives at the node from the the channel. Despite the favorable conditions for increase of the amount of substance $B$ the presence of possibility for conversion of $B$ to $A$ leads to a result that $B$ decreases to very low values after some time - Fig. 4a. Even if the conversion rate decreases and even if the amount of substance $B$ becomes larger than the amount of substance $A$ (Fig. 4b) the final result may be the same. Further decreasing of the value of $q$ may lead to large time of dominance of the substance $B$ in the node of the network - Fig. $4 \mathrm{c}$ but the final result can be the same as in the other two figures: because of the conversion $A$ prevails and $B$ is reduced to negligible amounts. Additional decreasing of the value of $q$ can however lead to change of the situation. At some critical low value of $q$ the conversion can't compensate anymore the rate of increase of $B$ and the amount of $B$ can exceed $A$. This dominance of the amount of substance $B$ can last as long as the parameters of the system in the studied node of the system remain unchanged. Such a situation can be easily observed if we just put $q=0$ in Eqs.(19). There is no more coupling of the amounts of the substances $A$ and $B$ and because of its larger rate of increasing it is just a matter of time for the amount of substance $B$ to exceed the amount of substance $A$. All this shows that the mechanism of limitation by conversion has its limits.

The combination of appropriate values of parameters can lead to interesting evolution of the amounts of the the substances $A$ and $B$ in the studied node of the network. Fig. 5 shows the influence of negative rate of increase of the substance $A$ compensated by positive rate of conversion from substance $B$ to substance $A$. The scenario here is Scenario No.2: Cyclic behavior of the amounts of the substance $A$ and $B$. The value of the rate of increase $r$ of substance $B$ increases from Fig. 5a to Fig. $5 \mathrm{c}$. The result is a cyclic evolution of the amounts of substances $A$ and $B$ and the period of the cycle is influenced by the parameter $r$ : an increase of $r$ leads to a decrease of the value of the period.

The parameter $c$ (it regulates the inflow of substance $B$ from the the channel to the studied node of the network) can have considerable influence on the dynamics of the amounts of substances $A$ and $B$ in the studied node of the network. One scenario: Scenario No.3: Dominance through inflow and conversion of substance connected to such a large influence is shown in Fig.6. Fig. 6a shows the situation in presence of a negligible amount of substance $B$ coming from the channel. The inflow of substance $B$ in not felt and the main processes that determine the dynamics of the substances in the node are the conversion of $B$ to $A$, the decrease of $A$ (e.g., because of its use in some process) and the increase of the amount of $B$ due to processes happening 

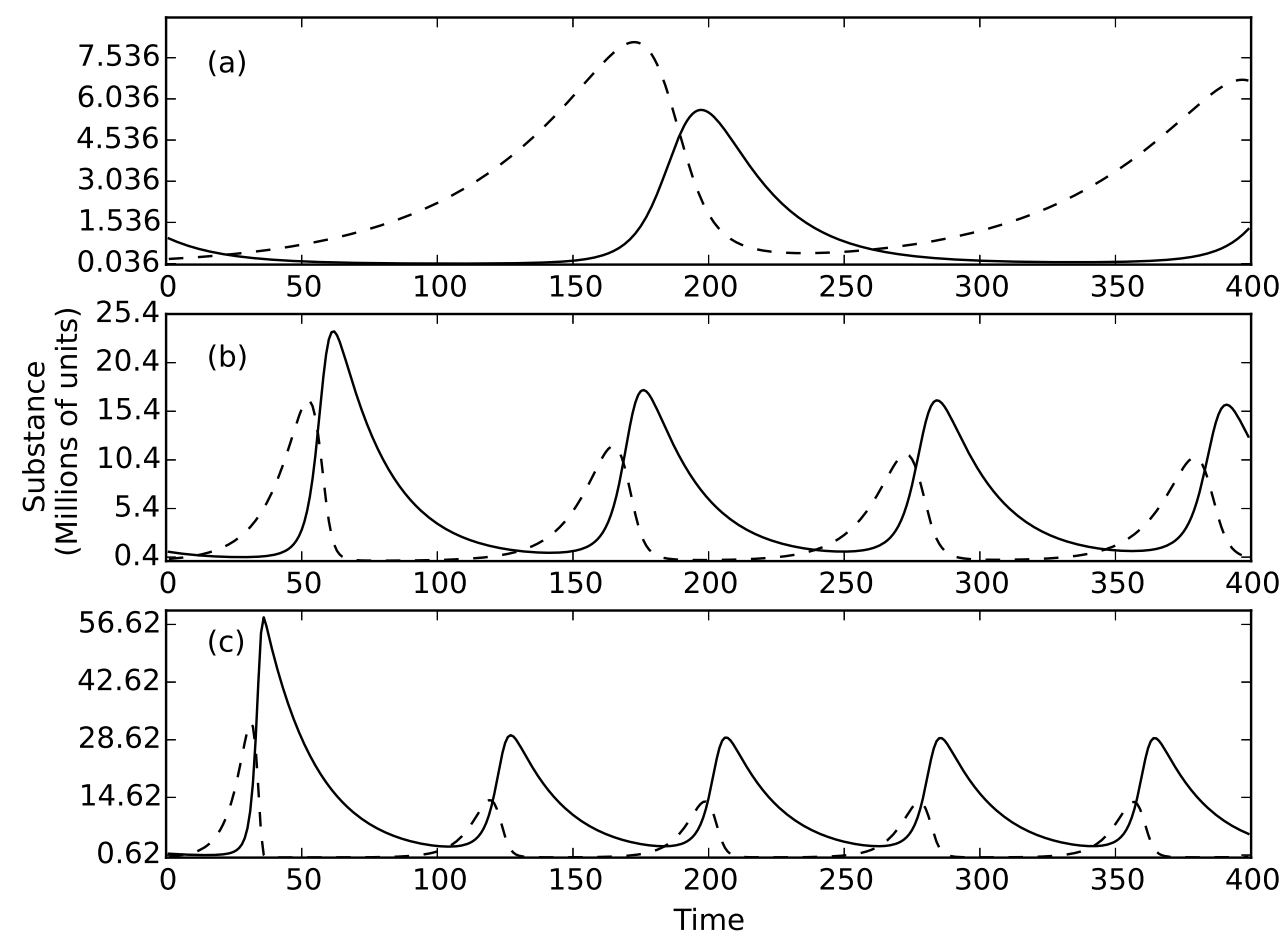

Figure 5: Interplay of conversion and negative rate $p$ of increase of the substance $A$ with the influence of increasing positive rate $r$ of increase of the amount of the substance $B$. Solid line: amount of substance $A$. Dashed line: amount of substance $B$. Initial conditions are: $A(0)=10^{6}, B(0)=2 \cdot 10^{5}$. The values of the parameters are as follows. $p=-0.05, q=2 \cdot 10^{-8}, c=5000$. Figure (a): $r=0.02$. Figure (b): $r=0.1$. Figure (c): $r=0.2$. The rate of (non-conversion) increase of the amount of substance $B$ influences the cyclic behavior of the substances $A$ and $B$.

inside the studied node of the network. As we can see a cyclic behavior of the amounts of substances occurs in the studied node. Let us now begin to increase the parameter $c$, i.e., the amount of substance $B$ per unit time increases that flows in the studied node from the channel. The characteristics of the cyclic behavior of the amounts of the substances in the studied node change. In Fig $6 \mathrm{~b}$ the value of $c$ is 750 time larger that the value of $c$ for Fig 6a. The period of the observed cycle decreases. The explanation is that the fast increasing of the substance $B$ activates the processes that convert substance $B$ to substance $A$ and these processes are much more intensive in 


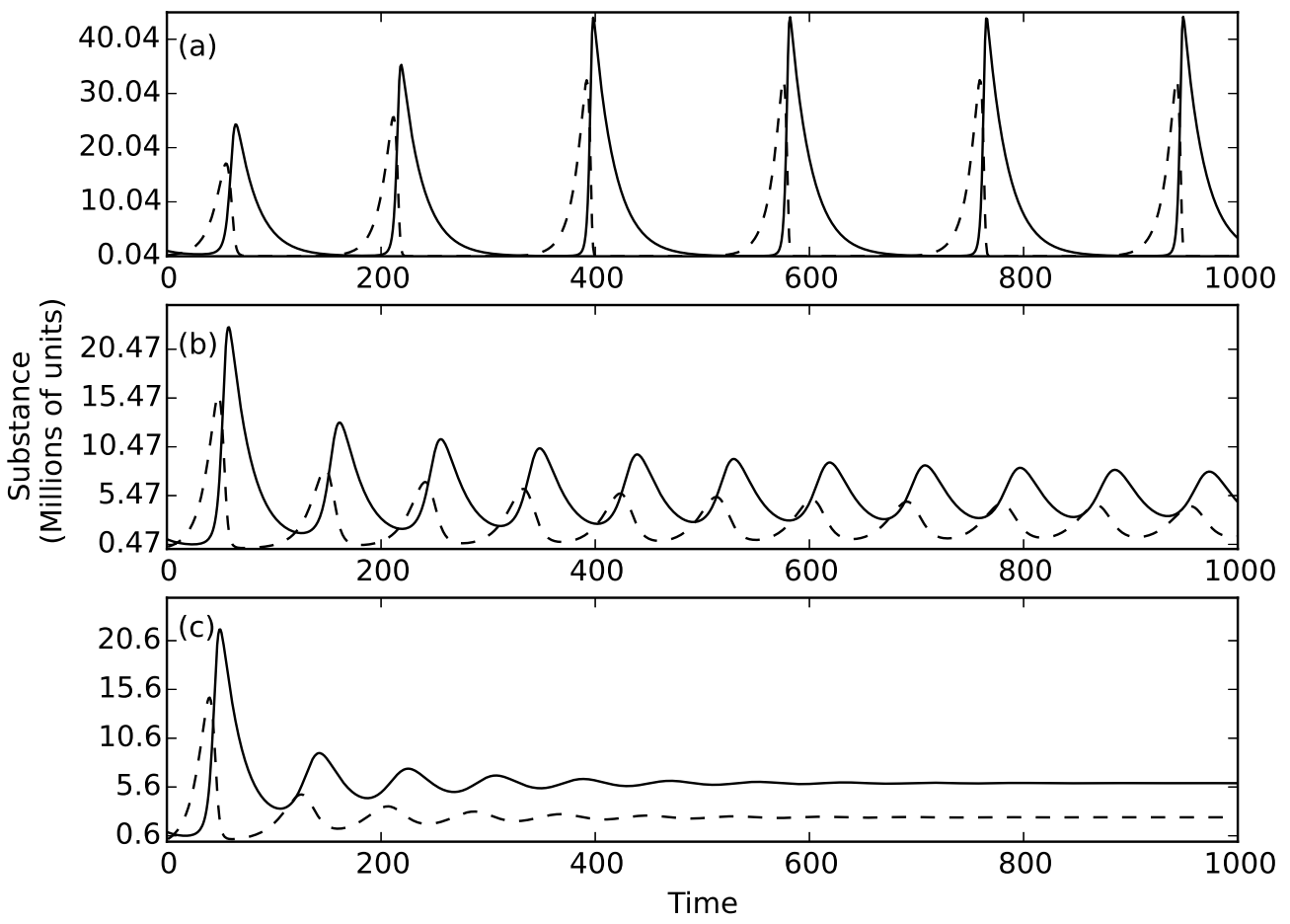

Figure 6: Influence of increasing value of parameter $c$ on the dynamics of the amounts of substances $A$ and $B$ in the studied node of the network. Interplay of conversion and negative rate of increase of the substance $A$ with the influence of increasing positive rate of increase of the amount of the substance $B$. Solid line: amount of substance $A$. Dashed line: amount of substance $B$. Initial conditions are: $A(0)=10^{6}, B(0)=2 \cdot 10^{5}$. The values of the parameters are as follows. $p=-0.05, q=2 \cdot 10^{-8}, r=0.1$. Figure (a): $c=20$. Figure (b): $c=15,000$. Figure (c): $c=50,000$.

comparison to the case shown in Fig. 6a. Further increasing of the value of $c$ (further increasing of the amount of inflow of substance $B$ from the channel to the node of the network) leads to vanishing of the cyclic behavior - Figure 6c. Instead of this a stationary state occurs where the amount of the substances $A$ and $B$ in the studied node have constant values.

The last situation we shall discuss for the case of constant values of the parameters in the model equations is the situation of positive $p$ (the processes in the node of the network lead to increasing amount of the substance $A$ ), negative $r$ (the processes in the node of the network lead to decreasing 

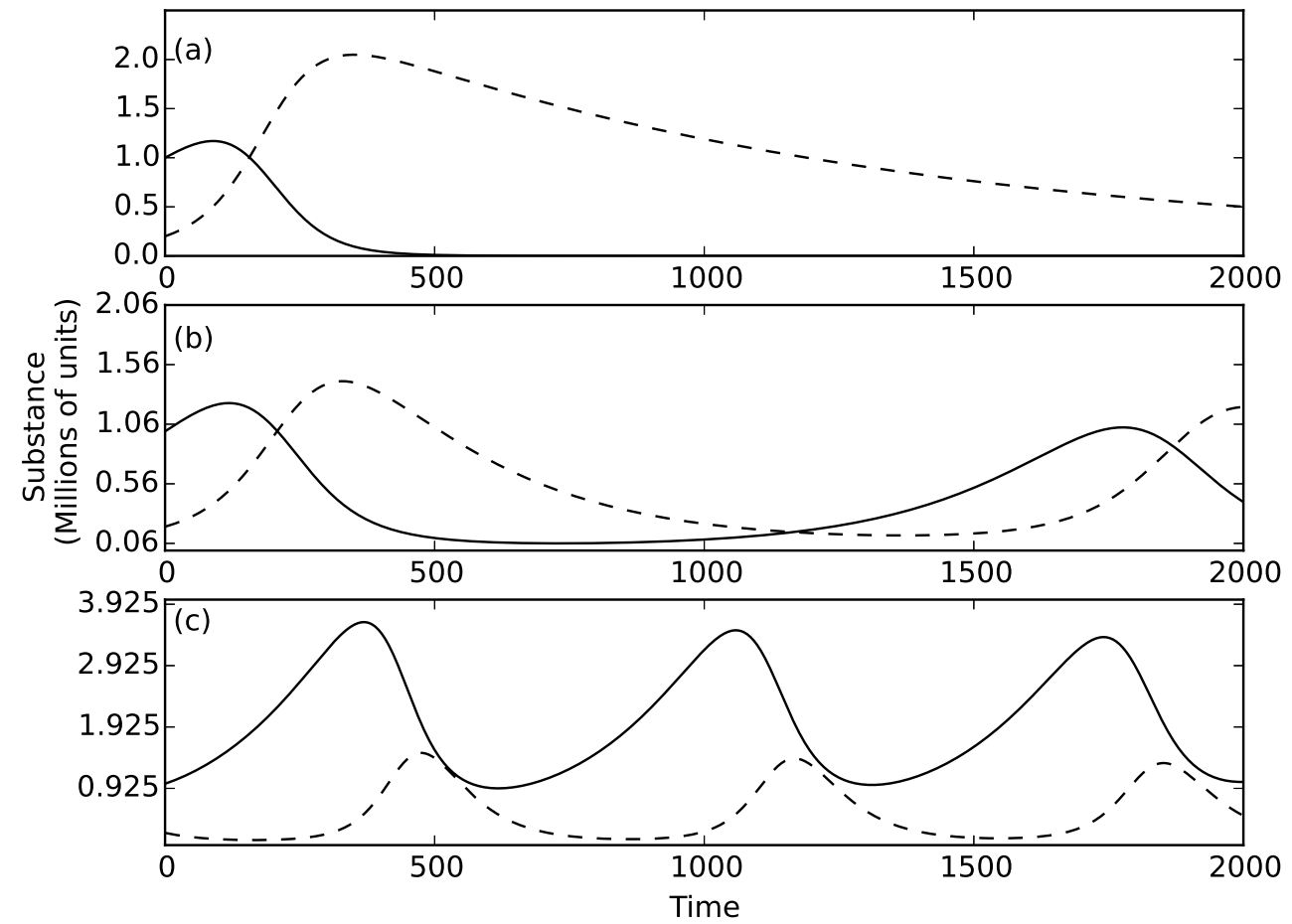

Figure 7: Influence of negative value of parameter $r$ and negative value of parameter $q$ on the dynamics of the amounts of substance in the studied node of the network. Solid line: amount of substance $A$. Dashed line: amount of substance $B$. Initial conditions are: $A(0)=10^{6}, B(0)=2 \cdot 10^{5}$. The values of the parameters are as follows. $p=0.005, q=-10^{-8}, c=100$. Figure (a): $r=-0.001$. Figure (b): $r=-0.004$. Figure $(\mathrm{c}): r=-0.02$.

amount of the substance $B$ ) and negative values of $q$ (processes happen in the network node that lead to conversion of substance $A$ to substance $B$ ) - Scenario No.4: Conversion can't compensate for decreasing. Fig.7 shows the influence of decreasing values of the rate $r$. Large values of $r$ lead to dominance of the substance $B$ : despite the smaller value of this substance at the node of the network in the initial moment of time the amount of the substance $B$ increases fast because of the conversion and then remains larger that the amount of the substance $A$ (and this is despite the fact that the rate $p$ is positive). The decreasing of the value of the rate $r$ leads to appearing of cyclic behavior and there is an interval of values of the rate $r$ where the dominance is exchanged: for some time interval the amount of the substance 
$B$ is larger than the amount of the substance $A$ and in the next time interval the amount of the substance $A$ is larger that the amount of the substance $B$. If the value of the ratio $r$ decreases further then the cyclic behavior in the node may persist for the long time but the substance $A$ remains dominant. At some value of $r$ the cyclic behavior vanishes. The vanishing may take some time (as in the case of Fig. 6c). or may be faster if the value of $r$ is small enough.
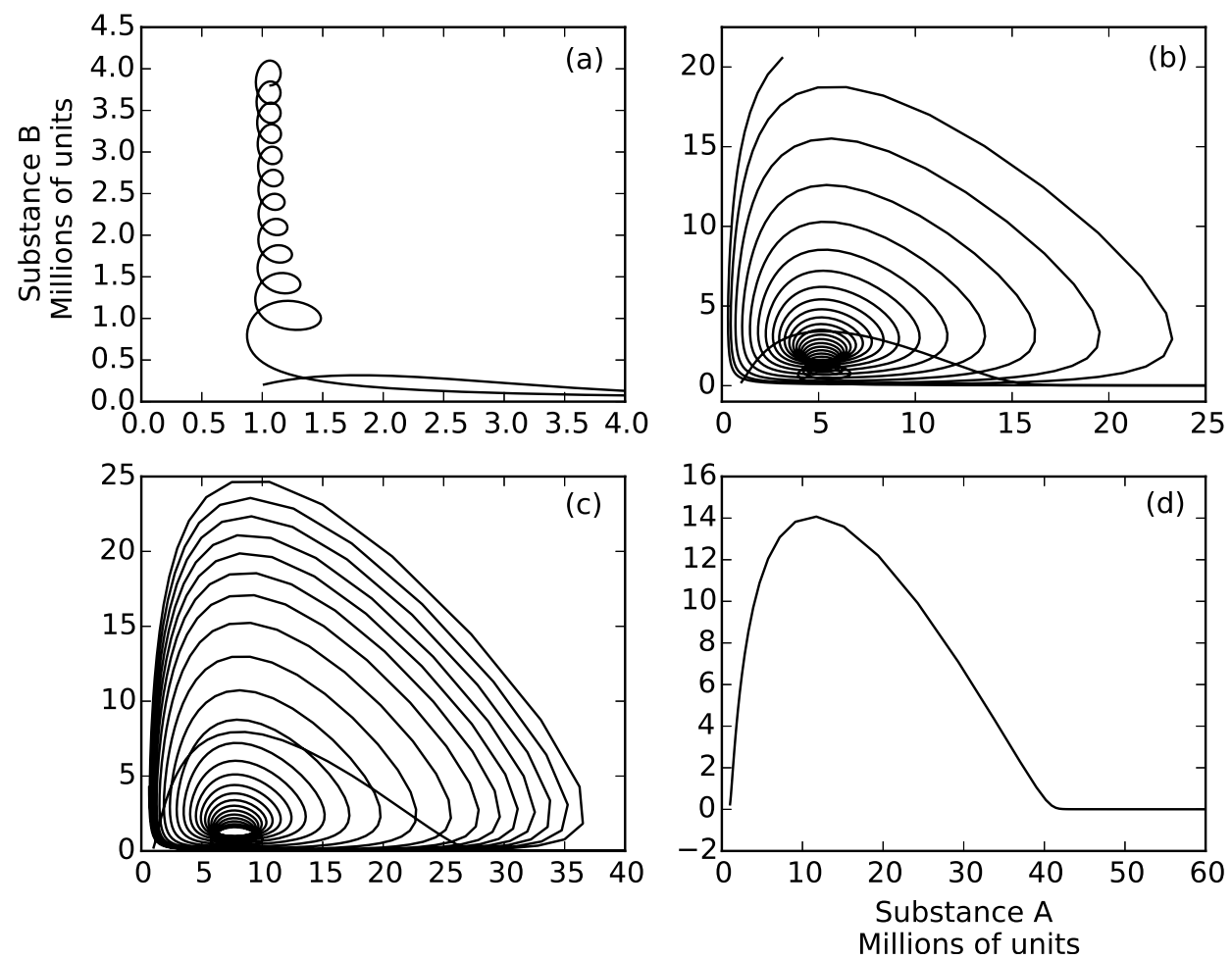

Figure 8: Evolution of the system for the case of coefficient $p\left(t_{k}\right)$ that decreases with increasing time. On all figures $p\left(t_{k}\right)=0.01-0.00003 t_{k}$. The other parameters of the system (19) are: $q=2 \cdot 10^{-8}, c=5000$. Parameter $r$ has different vales as follows. Figure (a): $r=0.02$. Figure (b): $r=0.1$. Figure (c): $r=0.15$. Figure (d): $r=0.2$ The initial values for the amounts of the substance are: $A(0)=10^{6}, B(0)=2 \cdot 10^{5}$.

Let us now discuss Eqs.(19) for some cases when the participating parameters change their values in the course of the time. If Figs. 8 and 9 we present results for such situation in which the parameter $p$ decreases slowly with the time for some finite interval of time. This decrease is the same for Figs. 8a 
- 8d. In addition we have different values of parameter $r$ : the smallest value of $r$ is for the situation shown in Fig.8a and the largest value of $r$ is for the situation shown in Fig. 8d. Two oscillation regimes are observed. Let us call them regime of smaller amplitude oscillations and regime of larger amplitude oscillations. The regime of smaller amplitude oscillations is shown in Fig. 8a. The specific feature of this regime is that the amount of the substance $A$ oscillates in time around a fixed value whereas the amount of the substance $B$ oscillates in time around a line characterizing a trend of increase of the value of substance $B$. The increasing value of parameter $r$ decreases the interval of time in which the substances from the network node change their amount in the regime of smaller amplitude oscillations. This regime is followed by a regime of larger amplitude oscillations - Fig. 8b. Further increase of the value of $r$ leads to an increase of the time in which the system of two substances is in the regime of larger amplitude oscillations. Finally the increase of the value of $r$ above some threshold value leads to vanishing of the oscillation regime for the values of the substances - Fig. 8d.

Figure 9 is connected again to the situation of slowly decreasing values of the parameter $p$ with increasing time. In Figs. 9a - 9d this decrease is the same. The differences among the figures $9 \mathrm{a}-9 \mathrm{~d}$ are because of the differences in the values of the parameter $q$. The smallest value of $q$ is for Fig. 9a and the largest value of $q$ is for Fig. 9d. The values of parameters in Fig. 9a lead to a non-oscillation regime for the both substances in the cell of the network. The increase of conversion rate $q$ leads to arising of a regime of larger oscillations - Fig. $9 \mathrm{~b}$ and with further increasing value of $q$ one observes regime of smaller amplitude oscillation of the amount of the substances that is followed by a regime of larger amplitude oscillations - Fig. 9c. The increasing of the value of parameter $q$ above a threshold value leads to vanishing of the oscillation regime. Thus for the case of slowly decreasing of the value of $p$ in the time the effect of the increasing $q$ is opposite to the effect of increasing $r$.

\section{Application of obtained results to the case of motion of migrants in a migration chan- nel}

Let us now consider the models discussed above from the point of view of: (i) dynamics of motion of migrants in a migration channel and (ii) dynamics of migrant population $B$ and native population $A$ in a corresponding node (country) of the network of countries. Let us remember that from the point of 

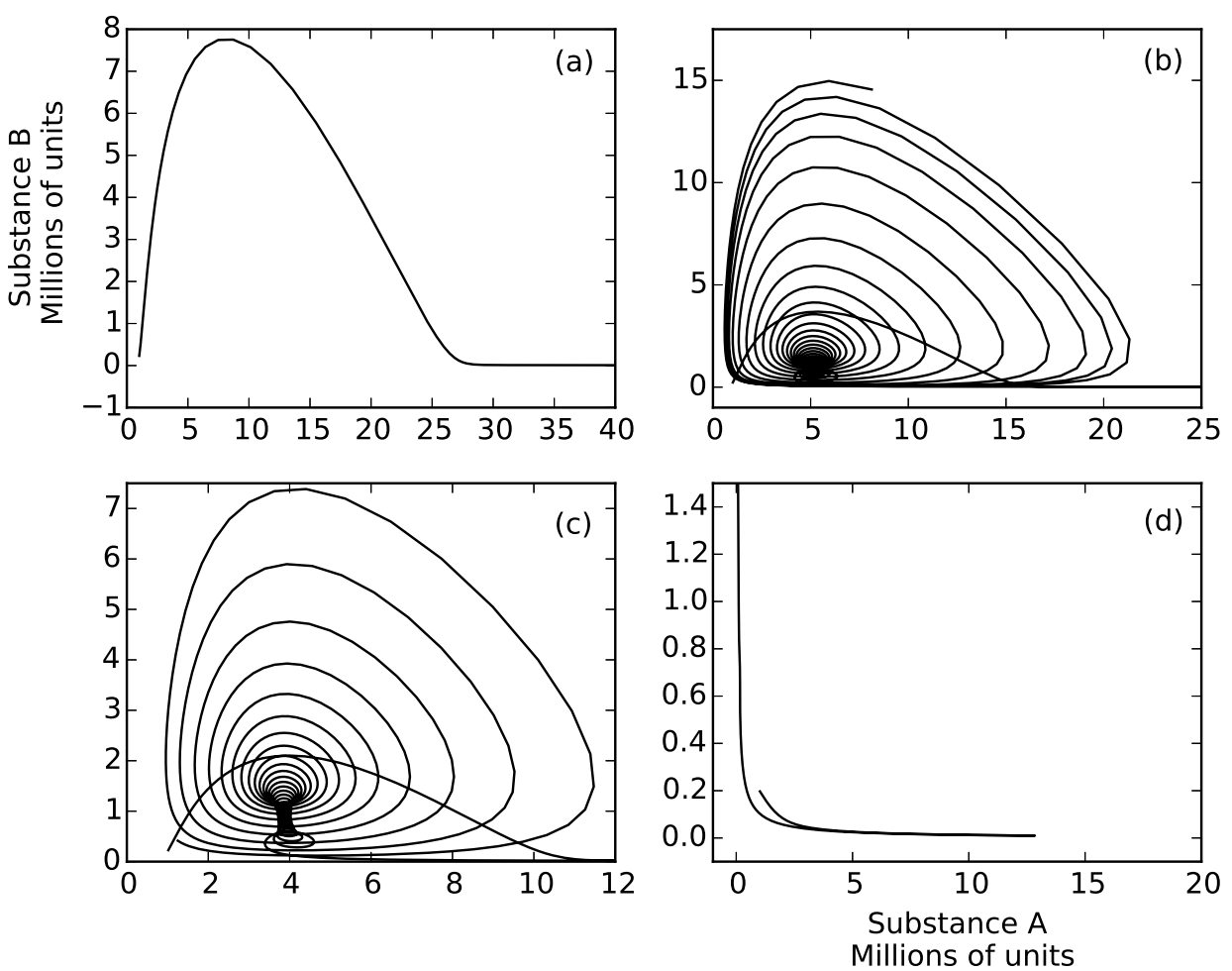

Figure 9: Evolution of the system for the case of coefficient $p\left(t_{k}\right)$ that decreases with increasing time. On all figures $p\left(t_{k}\right)=0.011-0.00003 t_{k}$. The other parameters of the system (19) are: $r=0.15, c=5000$. Parameter $q$ has different vales as follows. Figure (a): $q=2 \cdot 10^{-8}$. Figure (b): $q=3 \cdot 10^{-8}$. Figure (c): $q=4 \cdot 10^{-8}$. Figure (d): $q=5 \cdot 10^{-8}$. The initial values for the amounts of the substance are: $A(0)=10^{6}, B(0)=2 \cdot 10^{5}$.

view of modeling of migration flows we shall consider the channel described in the previous sections as a chain of countries. Migrants enter the channel from the entry country and may move through the channel to its last node (the last country called final destination country). In the general case migrants may move in the both directions: towards the final destination country or towards the entry country of the channel. The "leakage" in the channel is connected to change of the status of some migrants, e.g., they may obtain permission to stay in the corresponding country. This leakage may lead to situation in which one may observe a presence of native population $A$ and population of migrants $B$ in a country. These populations have growth rates $(p$ and $r)$. In general $p$ and $r$ depend on the time. In addition a process of integration 
may exist: the migrants of the population $B$ become native citizens in the course of the time (case of positive values of the parameter $q$ ). $q$ may have also non-positive values, i.e., the migrants are not integrated and they may start to convert the native population that accepts the characteristics of the migrant population. Then instead of integration one may observe absorption of the native population by the population of migrants.

Let us first discuss Figs. 2 and 3 from the point of view of migration dynamics. We note that for the case of migration channel and for distributions where $f_{i}=\alpha_{i}+\beta_{i} i$, the parameters $\alpha_{i}$ characterize permeability of the the borders between the $i$-th and $i+1$-st country of the channel. The parameters $\beta_{i}$ characterize the attractiveness of the $i$-th country of the channel and the parameters $\gamma_{i}$ characterize the part of migrants that obtain permission to stay in the $i$-th country of the channel. For the cases visualized in Fig. 2 the "leakage" parameter $\gamma_{i}$ has the same values for all four figures and for all countries of the channel. For the situation corresponding to Fig. 2a the permeability of the borders decrease with increasing $i$ and the attractiveness of the countries of the channel is the same. The form of the distribution (16) is similar to the standard form of such distribution for the case of infinite channel (see Appendix A). Specific effect connected to the finite channel arises in Fig. 2b. The situation there is characterized by increasing permeability of the borders between the countries of the channel with increasing value of $i$ and by lack of attractiveness of the countries of the channel $\left(\beta_{i}=0\right)$. In this case the probability connected with the final destination country is larger than the probabilities connected to countries of the channel with smaller value of $i$. This corresponds to concentration of migrants in the final destination country of the channel. Let us call this effect CP effects - effect of Concentration because of Permeability of the borders. Fig. 2c shows that $\mathrm{CP}$ effect can exist even if the attractiveness of the countries decreases with increasing value of $i$. Fig. 2d shows that CP effect can lead to unusual form of the distribution of migrants where the probability increases with increasing $i$ (this is opposite to the usual cases shown in Fig. 2a where probability decreases with increasing value of $i$ ).

Fig. 3 demonstrates another kind of effect we shall call CA effect - effect of Concentration because of Attractiveness. In all figures 3a, 3b, 3c, and $3 \mathrm{~d}$ the value of the "leakage parameter" $\gamma_{i}$ is the same for all countries of the channel. The same is the situation with the value of the parameter $\alpha_{i}$. From Figs. 3a and 3b we observe the CA effect - if we set the attractiveness parameter $\beta_{i}$ to 0 then the large probability for the last country of the channel vanishes - Fig. 3b. This clearly shows the existence of CA effect. Thus we have two effects that influence the number of the migrants in the last country of the channel (the final destination country) - CP effect and CA effect. Fig. 
3d shows that $\mathrm{CA}$ effect can be quite large if the parameter $\beta$ has large enough positive vales.

Figures 4 - 9 describe the dynamics of native and migrant populations in a country in presence of inflow of migrants from a migration channel. We note that this situation may become more actual in the course of years as the human population of Earth still increases and there are numerous military conflicts accompanied by poverty in many countries. In addition the climate changes may lead to large additional migration ("climate" migration). Above we have described 4 scenarios and let us now discuss these scenarios from the point of view of dynamics of human migration. Fig. 4 shows the effect of integration of the migrants in the corresponding society. If the integration politics is consequent it can lead to increase of the native population in the course of the time even if the rate of increase of the native population (accounted by the parameter $p$ ) are much smaller than the rate of increase of the migrant population (accounted by the parameter $r$ ). Of course the capabilities of integration are limited. If the inflow of migrants is large and the integration is not effective it is a matter of time for the situation to happen in which the number of non-integrated migrants will be larger and then much larger that the number of people from the native population.

Fig. 5 describes another possible scenario connected to a cyclic behavior of the number of individuals from native and migrant populations. This situation may arise when the rate of increase of the native population (births minus deaths) is negative and the rate of conversion is positive (a number of migrants are integrated and become part of the native population). The period of the cycle depends on the rate $r$ of increase of the population of migrants. With increase of $r$ the period of domination of migrants population (the time in which the number of migrants is large than the number of individuals from the native population) decreases. What we observe is a possibility of large intervals of time characterized by dominance of the migrant population. It may happen that the sign of the parameter $q$ is reversed at some moment of such an interval. Then the native population of the country may become extinct. Another scenario is shown in Fig. 6. Here despite (i) the increasing inflow of migrants from the migration channel and (ii) a positive values of the parameter $r$, stable dominance of the native population exists. The reason for this is the integration politics leading to positive value of the parameter $p$.

An interesting effect of negative value of the parameter $q$ (case when migrants integrate the native population) and negative value of parameter $r$ (the rate of increasing of migrants (births minus deaths) is negative) is shown in Fig. 7. When $r$ has small negative values the interval of time of domination of the migrant population can be quite large - Fig. 7a. When 
parameter $r$ decreases however the native population can become dominant despite the situation with the integration and a cyclic behavior occurs for the numbers of populations of migrants and native individuals.

The time-dependence of the parameters of the model of native and migrant populations leads to more complicated dynamics. Two possible scenarios are shown in Figs. 8 and 9. We note the following: there is a specific diagonal in all cases of Figs. 8 and 9. This diagonal starts from the bottom left corner of the corresponding figure and ends at the top right corner of the figure. If the phase trajectory is below this diagonal then there is a dominance of the native population (the number of individuals from the native population is larger than the number of individuals of the migrants population). If the phase trajectory is above this diagonal then the population of migrants is dominant. In Fig. 8a we observe a regime of small oscillations that happens in the area of dominance of migrants population. The increase of the value of parameter $r$ leads to substitution of this regime by regime of large amplitude oscillations - Figs. 8b, 8c. Finally a regime of dominance of population $A$ occurs - Fig. 8d. Fig. 9 shows several possible situations for the case when the rate $p\left(t_{k}\right)$ of increasing of the native population decreases slowly and in addition the rate of increasing of the migrant population increases from Fig. 9a to Fig. 9d. There is a constant rate of conversion of migrant population to native population. The final results from this situation may vary: domination of the native population - Fig. 9a; large oscillations of the number of individuals with exchange of domination - Fig. 9b; small oscillations with dominance of migrant population - Fig. 9c; final domination of the native population - fig. 9d.

\section{Concluding remarks}

In this article we discuss a discrete-time model of motion of substance in a finite-size channel of a network. The mathematical form of the general model is given by Eqs. (1) - (3). The particular case where the exchange of substance between the channel and the environment happens only through first node of the channel is studied in more detail. For the stationary regime of motion of a substance through the channel we obtain a new class of statistical distributions that contain as particular case the truncated Waring distribution. Further we study the dynamics of two substances in a node of network that has access to the channel and because of this some amount of substance leaks from the channel to the studied node of the network. The second substance is specific for the node of the network and can interact with the substance that comes from the channel. Four possible scenarios for the 
dynamics of the amounts of substance are described. The obtained results for the general case of motion of substance through the channel are applied to the case of motion of migrants in a migration channel that is positioned in a network of countries. Finally in Appendix A we obtain the class of statistical distributions for the case of stationary motion of substance in a channel of infinite length. These distributions contain as particular cases the famous long tail Waring distribution, Yule-Simon distribution and Zipf distribution.

\section{A Statistical distribution of the substance for the case of channel of network containing infinite number of nodes}

Let us consider the system of model equations for the case of infinite channel that corresponds to the system of model equations (11)-(13) for the case of finite channel. This system is

$$
\begin{gathered}
x_{0}\left(t_{k+1}\right)=x_{0}\left(t_{k}\right)+\sigma x_{0}\left(t_{k}\right)-f_{0} x_{0}\left(t_{k}\right)-\gamma_{0} x_{0}\left(t_{k}\right) \\
x_{i}\left(t_{k+1}\right)=x_{i}\left(t_{k}\right)+f_{i-1} x_{i-1}\left(t_{k}\right)-f_{i} x_{i}\left(t_{k}\right)-\gamma_{i} x_{i}\left(t_{k}\right) \quad i=1,2, \ldots
\end{gathered}
$$

Let us now consider a stationary state: $x_{i}\left(t_{k}\right)=x_{i}^{*}$. This stationary state occurs when $x_{i}\left(t_{k+1}\right)=x_{i}\left(t_{k}\right)$. From the system of equations (21), (22) we obtain $\left(i=1,2 \ldots, x_{0}^{*}\right.$ is a free parameter $)$

$$
x_{i}^{*}=x_{0}^{*} \prod_{j=1}^{i} \frac{f_{j-1}}{f_{j}+\gamma_{j}}
$$

The total amount of the substance in the channel is

$$
x^{*}=x_{0}^{*}\left[1+\sum_{k=1}^{\infty} \prod_{j=1}^{k} \frac{f_{j-1}}{f_{j}+\gamma_{j}}\right]
$$

We can consider the statistical distribution of the amount of substance along the nodes of the channel $y_{i}^{*}=x_{i}^{*} / x^{*}$. For this distribution we obtain

$$
\begin{aligned}
y_{0}^{*} & =\frac{1}{\left[1+\sum_{k=1}^{\infty} \prod_{j=1}^{k} \frac{f_{j-1}}{f_{j}+\gamma_{j}}\right]} \\
y_{i}^{*} & =\frac{\prod_{j=1}^{i} \frac{f_{j-1}}{f_{j}+\gamma_{j}}}{\left[1+\sum_{k=1}^{\infty} \prod_{j=1}^{k} \frac{f_{j-1}}{f_{j}+\gamma_{j}}\right]} ; i=1,2, \ldots
\end{aligned}
$$


Eq.(25) describes a class of statistical distributions $\left(f_{i}\right.$ and $\gamma_{i}$ are still not specified). To the best of our knowledge this general form of the class of distributions was not discussed by other authors. Fig. 10 shows that the
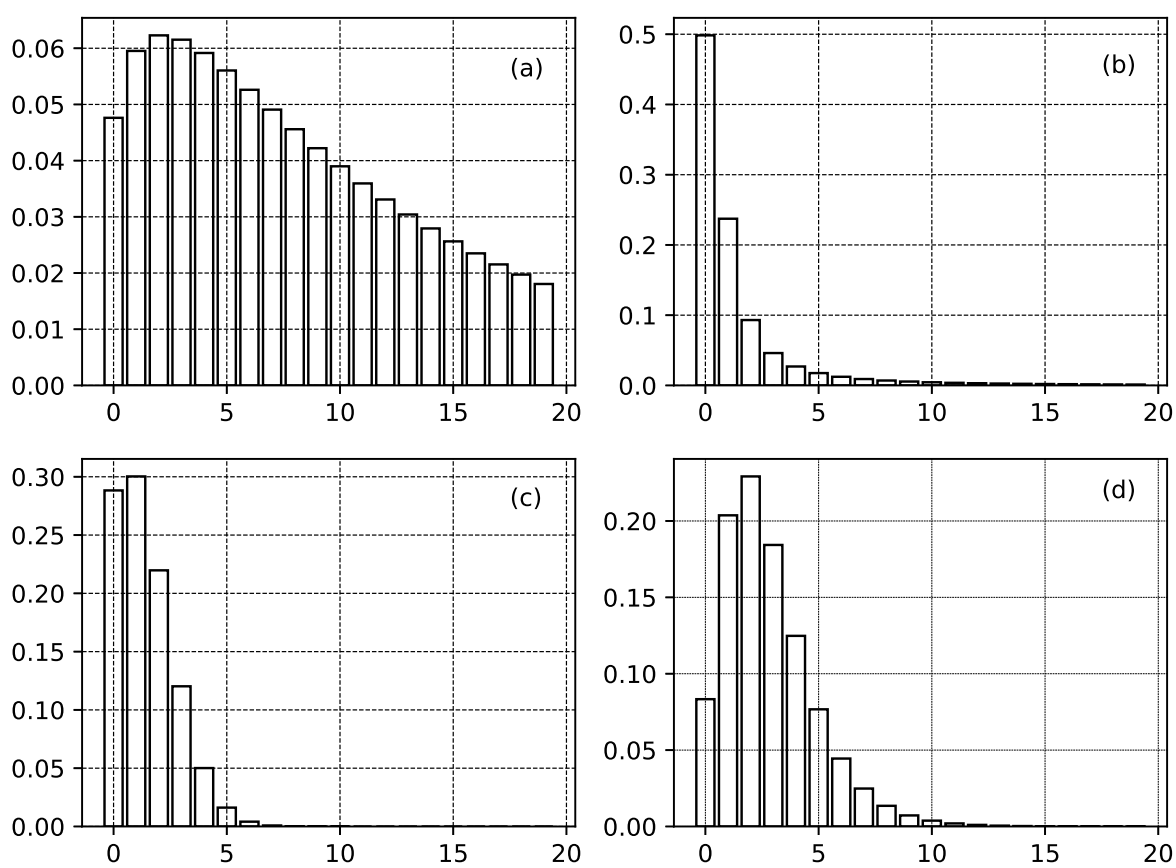

Figure 10: The distribution (25).$f_{i}$ is selected to be of the kind: $f_{i}=\alpha_{i}+i \beta_{i}$ where $\alpha_{i}$ and $\beta_{i}$ in general can depend on $i$. Figure (a): $f_{i}=0.001 /(i+$ $1)+0.001, \gamma_{i}=0.0001$. The effect of decreasing $\alpha_{i}$ with increasing $i$ is shown. Figure (b): $f_{i}=0.001+0.001 i^{2}, \gamma_{i}=0.001$. The effect of increasing $\beta_{i}$ with increasing $i$ is shown. Figure $(\mathrm{c}): f_{i}=0.001 /(i+1)+0.0015$, $\gamma_{i}=0.001(i+1)^{2}$. The effect of decreasing $\alpha_{i}$ and increasing $\gamma_{i}$ is shown. Figure $(\mathrm{d}): f_{i}=0.001 /\left((i+1)^{2}\right)+0.0001, \gamma_{i}=0.0001$. The effect of fast decreasing $\alpha_{i}$ with increasing $i$ is shown.

shapes of the distribution depends on the form of $f_{i}$ and $\gamma_{i} . f_{i}$ is of the kind $f_{i}=\alpha_{i}+i \beta_{i}$. This can be interpreted as $\alpha_{i}$ accounting for the permeability of the edge between $i$-th and $i+1$-th node of the channel and $\beta_{i}$ accounting for the "attractiveness" of the $i$-th node for the substance. Fig.10a shows that the decreasing permeability of edges of the channel may lead to increase of the amount of substance around the entry node and then the amount of substance 
decreases smoothly in the depth of the channel. When the permeability decreases very fast - Fig. 10d then the substance may concentrate in several nodes close to the entry node of the channel. Figure. 10b shows the influence of increasing "attractiveness" on the shape of the distribution: we observe the form of a standard long tail distribution. Figure. 10c shown the effect of decreasing permeability and increasing $\gamma_{i}$ which accounts for the "leakage" of substance in the corresponding node of the channel.

We note that the class of distributions (25) has interesting particular cases that have been discussed in connection with channels of migration of substance or migration channels of human migration. For and example let us consider the case where $\alpha_{i}$ and $\beta_{i}$ don't depend on $i, f_{i}=\alpha_{i}+\beta_{i} i$, $i=1, \ldots, N, \alpha_{i}>0, \beta_{i} \geq 0, \sigma_{0}>0, \gamma_{i} \geq 0$ (distributions of this kind have been discussed in [78] - [?]) . For this case the stationary distribution $x_{i}^{*}$ of the substance along the modes of the channel is given by the relationship

$$
x_{i}^{*}=\frac{\prod_{j=1}^{i}\left[\alpha_{i-j}+(i-j) \beta_{i-j}\right]}{\prod_{j=1}^{i}\left(\alpha_{j}+j \beta_{j}+\gamma_{j}\right)} x_{0}^{*}, i=1,2, \ldots
$$

and the statistical distribution connected to this stationary state of functioning of the channel is

$$
\begin{aligned}
& y_{0}^{*}= \frac{1}{1+\sum_{i=1}^{\infty} \frac{\prod_{j=1}^{i}\left[\alpha_{i-j}+(i-j) \beta_{i-j}\right]}{\prod_{j=1}^{i}\left(\alpha_{j}+j \beta_{j}+\gamma_{j}\right)}} \\
& y_{i}^{*}=\frac{\frac{\prod_{j=1}^{i}\left[\alpha_{i-j}+(i-j) \beta_{i-j}\right]}{\prod_{j=1}^{i}\left(\alpha_{j}+j \beta_{j}+\gamma_{j}\right)}}{1+\sum_{i=1}^{\infty} \frac{\prod_{j=1}^{i}\left[\alpha_{i-j}+(i-j) \beta_{i-j}\right]}{\prod_{j=1}^{i}\left(\alpha_{j}+j \beta_{j}+\gamma_{j}\right)}}, i=1,2, \ldots
\end{aligned}
$$

The distribution (27) is a generalization, e.g., of the Waring distribution [79] as well as a generalization of one of distributions discussed in [80]. Several forms connected to this distribution are shown in Fig.11. As we can see the changes in the values of the parameters $\beta_{i}$ influence the form of the distribution. From the point of view of application of theory to the case of 

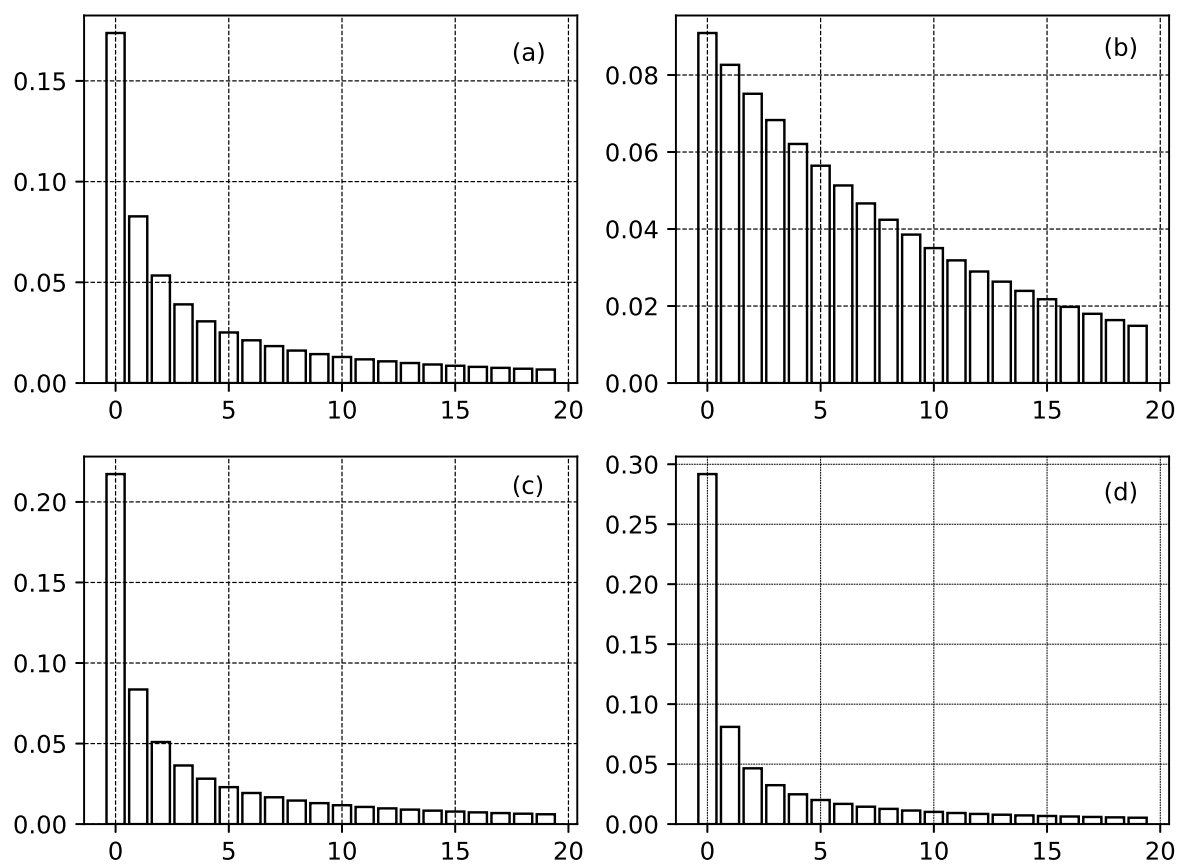

Figure 11: The distribution (27) for a channel of infinite length. All parameters $\gamma_{i}$ have the same value $\gamma_{i}=0.0001(i=0, \ldots$,$) in all figures -2 \mathrm{a}, 2 \mathrm{~b}$, 2c, 2d. All parameters $\alpha_{i}$ have the same value $\alpha_{i}=0.001(i=0, \ldots$,$) in all$ figures - 2a, 2b, 2c, 2d. Figures show the changes in the form of the distribution when the parameter $\beta_{i}$ is changed. Figure (a): $\beta_{i}=0.001 i$. Note that in the case of infinite channel the effect of concentration of the substance in the last node of the channel is missing. Figure (b): $\beta_{i}=0$. The amount of substance in the nodes of the network decreases smoothly from node to node of the channel. Figure (c): $\beta_{i}=0.0015 i . \beta_{i}$ are larger in comparison to Fig $2 \mathrm{a}$. The effect of these larger values of $\beta_{i}$ is concentration of substance in the entry node of the channel. This effect is seen also in Figure $(\mathrm{d})$ where the values of $\beta_{i}$ are even larger: $\beta_{i}=0.0025 i$.

channels of human migration parameters $\beta_{i}$ account for the attractiveness of the corresponding country of the channel. When all parameters $\beta_{i}=0$ (equally attractive countries) then the values of the distribution decrease slowly with increasing $i$. For the case of nonzero values of $\beta_{i}$ we observe increasing of the rate of migrants in the entry country of the channel. This 
effect is connected with the infinite size of the channel. If the chanel has a finite size then a concentration of substance in the last node of the channel could be observed.

\section{References}

[1] M. Ausloos, A. Gadomski, N. K. Vitanov. Primacy and ranking of UEFA soccer teams from biasing organization rules. Physica Scripta 89 (2014) 108002 .

[2] M. Ausloos, R. Cloots, A. Gadomski, N. K. Vitanov. Ranking structures and rankrank correlations of countries: The FIFA and UEFA cases. International Journal of Modern Physics C 25 (2014) 1450060.

[3] Z. Dimitrova, Z. On traveling waves in lattices: The case of Riccati lattices. Journal of Theoretical and Applied Mechanics 42 (2012) 3 - 22 (2012).

[4] Z. I. Dimitrova, K. N. Vitanov. Integrability of differential equations with fluid mechanics application: from Painleve property to the method of simplest equation. Journal of Theoretical and Applied Mechanics, 43 (2013) $31-42$.

[5] Z. I. Dimitrova. Numerical investigation Of nonlinear waves connected to blood flow in an elastic tube with variable radius. Journal of Theoretical and Applied Mechanics 45 (2015) 79 - 92.

[6] Z. I. Dimitrova, N. K. Vitanov. Influence of adaptation on the nonlinear dynamics of a system of competing populations. Physics Letters A 272 (2000) $368-380$.

[7] Z. I. Dimitrova, N. K. Vitanov. Dynamical consequences of adaptation of the growth rates in a system of three competing populations. Journal of Physics A: Mathematical and General 34 (2001) 7459 - 7473.

[8] Z. I. Dimitrova, N. K. Vitanov. Adaptation and its impact on the dynamics of a system of three competing populations. Physica A: Statistical Mechanics and its Applications 300 (2001) 91 - 115.

[9] Z. I. Dimitrova, N. K.Vitanov. Chaotic pairwise competition. Theoretical Population Biology 66 (2004) 1 - 12. 
[10] E. V. Nikolova, I. P. Jordanov, Z. I. Dimitrova, N. K. Vitanov. Nonlinear evolution equation for propagation of waves in an artery with an aneurysm: An exact solution obtained by the modified method of simplest equation. pp. 131 - 144 in K. Georgiev, M. Todorov, I. Georgiev (Eds.) Advanced Computing in Industrial Mathematics. Springer, Cham, 2018.

[11] H. Kantz, D. Holstein, M. Ragwitz, N. K. Vitanov. Markov chain model for turbulent wind speed data. Physica A: Statistical Mechanics and its Applications 342 (2004) 315 - 321.

[12] S. Panchev, T. Spassova, N. K. Vitanov. Analytical and numerical investigation of two families of Lorenz-like dynamical systems. Chaos, Solitons \& Fractals 33 (2007) 1658 - 1671.

[13] K. Sakai, S. Managi, N. K. Vitanov, K. Demura. Transition of chaotic motion to a limit cycle by intervention of economic policy: an empirical analysis in agriculture. Nonlinear dynamics, psychology, and life sciences 11 (2007) 253 - 265.

[14] N. K. Vitanov, Z. I. Dimitrova. Application of the method of simplest equation for obtaining exact traveling-wave solutions for two classes of model PDEs from ecology and population dynamics. Communications in Nonlinear Science and Numerical Simulation 15 (2010) 2836 - 2845.

[15] N. K. Vitanov, Z. I. Dimitrova. On waves and distributions in population dynamics. BIOMATH, 1 (2012) 1209253.

[16] N. K. Vitanov, N. P. Hoffmann, B. Wernitz. Nonlinear time series analysis of vibration data from a friction brake: SSA, PCA, and MFDFA. Chaos, Solitons \& Fractals, 69 (2014) 90 - 99.

[17] N. K. Vitanov, Z. I. Dimitrova, K. N. Vitanov. Modified method of simplest equation for obtaining exact analytical solutions of nonlinear partial differential equations: further development of the methodology with applications. Applied Mathematics and Computation 269 (2015) $363-378$.

[18] N. K. Vitanov, M. Ausloos. Test of two hypotheses explaining the size of populations in a system of cities. Journal of Applied Statistics, 42 (2015) $2686-2693$.

[19] N. K. Vitanov, Z. I. Dimitrova, T. I. Ivanova. On solitary wave solutions of a class of nonlinear partial differential equations based on the function 
$1 / \cosh ^{n}(\alpha x+\beta t)$. Applied Mathematics and Computation 315 (2017) $372-380$.

[20] R. Albert, A. -L. Barabasi. Statistical mechanics of complex networks. Rev. Mod. Phys. 74 (2002) 47 - 97.

[21] L. A. N. Amaral, J. M. Ottino. Complex networks. Augmenting and framework for the study of complex systems. Eur. Phys. J. B 38 (2004) $147-162$.

[22] S. Boccaletti, V. Latora, Y. Moreno, M. Chavez, D. U. Hwang. Complex networks: Structure and dynamics. Physics Reports, 424 (2006) 175 308.

[23] V. Petrov, E. Nikolova, O. Wolkenhauer. Reduction of nonlinear dynamic systems with an application to signal transduction pathways. IET Systems Biology, 1 (2007) $2-9$.

[24] N. K. Vitanov. Science dynamics and research production: Indicators, indexes, statistical laws and mathematical models. Springer, Cham, 2016.

[25] N. K. Vitanov, I. P. Jordanov, Z. I. Dimitrova. On nonlinear dynamics of interacting populations: Coupled kink waves in a system of two populations. Communications in Nonlinear Science and Numerical Simulation 14, (2009) 2379 - 2388.

[26] N. K. Vitanov, I. P. Jordanov, Z. I. Dimitrova. On nonlinear population waves. Applied Mathematics and Computation 215 (2009) 2950 - 2964.

[27] N. K. Vitanov, Z. I. Dimitrova, M. Ausloos. Verhulst-Lotka-Volterra model of ideological struggle. Physica A 389 (2010) 4970 - 4980.

[28] N. K. Vitanov, M. Ausloos, G. Rotundo Discrete model of ideological struggle accounting for migration. Advances in Complex Systems 15, Supplement 1 (2012) Article number 1250049.

[29] N. K. Vitanov, M. Ausloos. Knowledge epidemics and population dynamics models for describing idea diffusion. pp. 69 - 125 in Scharnhorst, A., Börner, K., van den Besselaar, P. (Eds.) Models of science dynamics, Berlin, Springer, 2012.

[30] N. K. Vitanov, Z. I. Dimitrova, K. N. Vitanov. Traveling waves and statistical distributions connected to systems of interacting populations. Computers \& Mathematics with Applications 66 (2013) 1666 - 1684. 
[31] N. K. Vitanov, K. N. Vitanov. Population dynamics in presence of state dependent fluctuations. Computers \& Mathematics with Applications 68 (2013) 962 - 971.

[32] N. K. Vitanov, M. Ausloos. Test of two hypotheses explaining the size of populations in a system of cities. Journal of Applied Statistics, 42 (2015) $2686-2693$.

[33] L. D. Ford, Jr., D. R. Fulkerson. Flows in networks. Princeton University Press, Princeton, NJ, 1962.

[34] G. Ruhe. Algorithmic aspects of flows in networks. Springer, Netherlands, 1991.

[35] R.K. Ahuja, T. L. Magnanti, J. B. Orlin. Network flows. Theory, algorithms, and applications. Prentice Hall, NJ, 1993.

[36] D. T. Duval. Tourism and transport. Modes, networks, and flows. Channel View Publications, Buffalo, Toronto, 2007.

[37] M. T. Todinov. Flow networks. Analysis and optimization of repairable flow networks, networks with disturbed flows, static flow networks and reliability networks. Elsevier, Amsterdam, 2013.

[38] V Tejedor, O Benichou, R Voituriez. Close or connected: Distance and connectivity effects on transport in networks. Phys. Rev. E 83 (2011) 066102 .

[39] W.-K. Chan. Theory of nets: Flows in networks. Wiley, New York, 1990.

[40] L. Ambrosio, A. Bressan, D. Helbing, A. Klar, E. Zuazua (Eds.). Modeling and optimisation of flows on networks. Springer, Heidelberg, 2010.

[41] N. H. Gartner, G. Improta (Eds.) Urban traffic networks. Dynamic flow modeling and control. Springer, Berlin, 1995.

[42] E. Bernard, L. Jacob, J. Mairal, J.-P. Vert. Efficient RNA isoform identification and quantification from RNA-Seq data with network flows. Bioinformatics 30 (2014) 2447 - 2455.

[43] M. Rossvall, A.C. Esquivel, A. Lancichinetti, J. D. West, R. Lambiotte. Memory in network flows and its effects on spreading dynamics and community detection. Nature Communications 5 (2014) Article No. 4630. 
[44] R. E. Gomori, T. C. Hu. Multi-terminal network flows. J. Soc. Indust. Appl. Math. 9 (1961) $551-570$.

[45] G. Masson, B. W. Jordan, Jr. Generalized multi-stage connection networks. Networks 2 (1972) 191 - 209.

[46] W. C. Jordan, M. A. Turnquist. A stochastic dynamic model for railroad car distribution. Transportation Science 17 (1983), 123 - 145.

[47] D. Bertsimas, M. Sim. Robust discrete optimization and network flows. Mathematical Programming 98 (2003) 49 - 71.

[48] J. E. Aronson. A survey of dynamic network flows. Annals of Operation Research 20 (1989) 1 - 66.

[49] D. Helbing, L. Buzna, A. Johansson, T. Werner. Self-organized pedestrian crowd dynamics: Experiments, simulations, and design solutions. Transportation Science 39 (2005) 1 - 24.

[50] M. Skutella. An introduction to network flows over time. pp. 451 - 482 in W. Cook, L. Lovasz, J. Vygen (Eds.) Research trends in combinatorial optimization. Springer, Berlin, 2009.

[51] M. Treiber, A. Kesting. Traffic flow dynamics: Data, models, and simulation. Springer, Berlin, 2013.

[52] A. V. Bozhenyuk, E. M. Gerasimenko, J. Kacprzyk, I. Naumovich. Flows in networks under fuzzy conditions. Springer International Publishing Switzerland, 2017.

[53] E. S. Lee. A theory of migration. Demography 3 (1966) 47 - 57.

[54] R. Armitage. Population projections for English local authority areas. Population Trends 43 (Spring) (1986) 31-40.

[55] I. Bracken, J. J. Bates. Analysis of gross migration profiles in England and Wales: some developments in classification. Environment and Planning A 15 (1983) 343-355.

[56] A. G. Champion, G. Bramley, A. S. Fotheringham, J. Macgill, P. H. Rees. A migration modelling system to support government decisionmaking. pp. 257-278 in J. Stillwell, S. Geertman (Eds.) Planning support systems in practice. Springer Verlag, Berlin, 2002. 
[57] J. R. Harris, M. P. Todaro. Migration, unemployment and development: A two-sector analysis. The American Economic Review 60 (1970) 126 142.

[58] J. H. Simon. The economic consequences of migration. The University of Michigan Press, Ann Arbor, MI, 1999.

[59] R. Skeldon. Migration and development: A global perspective. Routledge, London, 1992.

[60] D. S. Massey, J. Arango, G. Hugo, A. Kouaougi, A. Pellegrino, J. Edward Taylor. Theories of international migration: A review and appraisal. Population and Development Review 19 (1993) 431 - 466.

[61] W. J. Ethier. International trade and labor migration. The American Economic Review 75 (1985) 691 - 707.

[62] G. J. Borjas. Economic theory and international migration. International Migration Review 23 (1989) 457 - 485.

[63] J. T. Fawcet. Networks, linkages, and migration systems. International Migration Review 23 (1989) 671 - 680.

[64] D. T. Gurak, F. Caces. Migration networks and the shaping of migration systems. pp. 150 - 176 in M. M. Kitz, L. L. Lim, H. Zlotnik (Eds.) International migration systems: A global approach. Clarendon Press, Oxford, 1992.

[65] F. J. Willekens. Probability models of migration: Complete and incomplete data. SA Journal of Demography 7 (1999) 31 - 43.

[66] J. Ledent. Multistate life table: movement versus transition perspectives. Environment and Planning A 12 (1980) 533 - 562.

[67] F. Willekens. Models of migration observations and judgment. pp. 117 147 in J. Raymer, F. Willekens (Eds.) International migration in Europe: Data, models and estimates. Wiley, New York, 2008.

[68] H. -P. Blossfeld, G. Rohwer. Techniques of event history modeling: new approaches to casual analysis. Lawrence Erlbaum, New Jersey, 2002.

[69] H. P. Blossfeld, K. Golsch, G. Rohwer (Eds.). Event history analysis with Stata . Lawrence Erlbaum, New Jersey, 2007. 
[70] D. S. Hachen. The competing risk model. Sociological Methods and Research 17 (1988) 21 - 54.

[71] B. Singer, S. Spilerman. Mathematical representations of development. Theories. pp. 155 - 177 in J. R. Nesselroade, P. B. Baltes (Eds.) Longitudinal research in the study of behavior and development.Academic Press, New York, 1979.

[72] L. Collins. Industrial migration in Ontario: forecasting aspects of industrial activity through Markov chains. Statistics Canada, Ottawa, 1972.

[73] L. Collins. An introduction to Markov chain analysis. Headey Brothers Ltd., London, 1975.

[74] G. J. McLachlan, T. Krishnan. The EM algorithm and extensions. Wiley, New York, 1977.

[75] J. Raymer. The estimation of international migration flows: a general technique focused on the origin-destination association structure. Environment and Planning A 39 (2007) 985 - 995.

[76] M. J. Bierley, J. J. Forster, J. W. McDonald, P. W. F. Smith. Bayessian estimation of migration flows, pp. 149 - 174 in J. Raymer, F. Willekens, (Eds.). International migration in Europe: Data, models and estimates. Wiley, New York, 2008.

[77] M. J. Greenwood. Modeling migration, pp. 725 - 734 in K. KempLeonard, (Ed.) Encyclopedia of social measurement, vol. 2, Elsevier, Amsterdam, 2005.

[78] A. Schubert, W. Glänzel. A dynamic look at a class of skew distributions. A model with scientometric application. Scientometrics 6 (1984) 149 167.

[79] N. K. Vitanov, K. N. Vitanov. Box model of migration channels. Mathematical Social Sciences 80 (2016) 108 - 114.

[80] N. K. Vitanov, K. N. Vitanov, T. Ivanova. Box model of migration in channels of migration networks. p.p. 203 - 215 in K. Georgiev et al. (Eds.) Advanced Computing in Industrial Mathematics, Studeies in Computational Intelligence No. 728, Springer, Berlin, (2018)

[81] N. K. Vitanov, K. N. Vitanov. On the motion of substance in a channel of network and human migration. Physica A 490 (2018) 1277 - 1294. 